\title{
Continuation and bifurcation analysis of delay differential equations
}

\author{
Dirk Roose ${ }^{1}$ and Robert Szalai ${ }^{2}$ \\ 1 Department of Computer Science, Katholieke Universiteit Leuven, Belgium \\ ${ }^{2}$ Department of Engineering Mathematics, University of Bristol, United Kingdom
}

Mathematical modeling with delay differential equations (DDEs) is widely used in various application areas of science and engineering (e.g., in semiconductor lasers with delayed feedback, high-speed machining, communication networks, and control systems) and in the life sciences (e.g., in population dynamics, epidemiology, immunology, and physiology). Delay equations have an infinite-dimensional state space because their solution is unique only when an initial function is specified on a time interval of length equal to the largest delay. Consequently, analytical calculations are more difficult than for ordinary differential equations andnumerical methods are generally the only way to achieve a complete analysis, prediction and control of systems with time delays.

Delay differential equations are a special type of functional differential equation (FDE). In FDEs the time evolution of the state variable can depend on the past in an arbitrary way as long as the dependence is a bounded function of the past. However, DDEs impose a constraint on this dependence, namely that the evolution depends only on certain past values of the state at discrete times. (We do not consider here the case of distributed delay.) The delays can be constant or state dependent. The equations can also involve delayed values of the derivative of the state, which leads to equations of neutral type.

In this chapter we mainly discuss the simplest case, namely a finite number of constant delays. Specifically, we consider a nonlinear system of DDEs with constant delays $\tau_{j} \geqslant 0, j=1, \ldots, m$, of the form

$$
x^{\prime}(t)=f\left(x(t), x\left(t-\tau_{1}\right), x\left(t-\tau_{2}\right), \ldots, x\left(t-\tau_{m}\right), \eta\right),
$$

where $x(t) \in \mathbb{R}^{n}$, and $f: \mathbb{R}^{(m+1) n+p} \rightarrow \mathbb{R}^{n}$ is a nonlinear smooth function depending on a number of (time-independent) parameters $\eta \in \mathbb{R}^{p}$. We assume that the delays are in increasing order and denote the maximal delay by

$$
\tau=\tau_{m}=\max _{i=1, \ldots, m} \tau_{i}
$$


A solution segment is denoted by $x_{t}=x_{t}(\theta)=x(t+\theta) \in C, \theta \in[-\tau, 0]$. Here $C=C\left([-\tau, 0] ; \mathbb{R}^{n}\right)$ is the space of continuous functions mapping the delay interval into $\mathbb{R}^{n}$. For a fixed value of the parameter $\eta$, a solution $x(t)$ of (1) on $t \in[0, \infty)$ is uniquely defined by specifying a function segment $x_{0}$ as an initial condition. A discontinuity in the first derivative of $x(t)$ generally appears at $t=0$ and is propagated in time, even if $f$ and $\phi$ are infinitely smooth. However, the solution operator of (1) smooths the solution, meaning that discontinuities appear in higher and higher derivatives as time increases.

A DDE can be approximated by a system of ordinary differential equations (ODEs) and so standard numerical methods for ODEs could be used. However, to obtain an accurate approximation a high-dimensional system of ODEs is needed, and this leads to expensive numerical procedures. During the last decade, more efficient and more reliable numerical methods have been developed specifically for DDEs. In this chapter we survey those numerical methods for the continuation and bifurcation analysis of DDEs that are implemented in the software packages DDE-BIFTOOL [25, 26] and PDDE-ConT [73]. Where appropriate, we also briefly describe alternative numerical methods. Note that we do not discuss time integration of DDEs; for this topic see, e.g., [2] and $[6]$.

The structure of this chapter is as follows. In Sec. 1 we discuss numerical methods to compute the right-most characteristic roots of steady-state solutions. In Sec. 2 we describe collocation methods for computing periodic solutions and their dominant Floquet multipliers. Section 3 presents defining systems for codimension-one bifurcations of periodic solutions that allow one to compute the location of bifurcation points accurately. Computation of connecting orbits is discussed in Sec. 4 and of quasiperiodic solutions is discussed in Sec. 5. In Sec. 6 we briefly discuss how to deal with special types of DDEs, specifically, equations of neutral type and DDEs with statedependent delays. In Sec. 7 we discuss specific details of the software packages DDE-BIFTOOL and PDDE-Cont. Their functionality is illustrated in Sec. 8, where we present the bifurcation analysis of several DDE models of practical relevance. Finally, conclusions and an outlook can be found in Sec. 9.

\section{Stability of steady-state solutions}

In this and the next section we assume that the parameter $\eta$ is fixed and we omit it from the equations. A steady-state solution $x(t) \equiv x^{\star}$ of (1) satisfies the nonlinear system

$$
f\left(x^{\star}, x^{\star}, x^{\star}, \ldots, x^{\star}\right)=0 .
$$

The (local) stability of $x^{\star}$ is determined by the stability of (the zero solution of) the linearized equation

$$
y^{\prime}(t)=A_{0} y(t)+\sum_{j=1}^{m} A_{j} y\left(t-\tau_{j}\right),
$$


where $A_{j} \in \mathbb{R}^{n \times n}$ denotes the partial derivative of $f$ with respect to its $(j+1)$-th argument, evaluated at the steady-state solution $x^{\star}$. The linearized equation (3) is asymptotically stable if all its roots $\lambda$ of the characteristic equation

$$
\operatorname{det}\left(\lambda I-A_{0}-\sum_{j=1}^{m} A_{j} \mathrm{e}^{-\lambda \tau_{j}}\right)=0
$$

lie in the open left half-plane (i.e., $\operatorname{Re}(e(\lambda))<0)$; see, e.g., $[38,58,70]$. Equation (4) has an infinite number of roots $\lambda$, known as the characteristic roots. However, the number of characteristic roots with real part larger than a given threshold is finite. Hence, to analyse the stability of a steady-state solution, one must determine reliably all roots satisfying $\operatorname{Re}(e(\lambda)) \geqslant r$, for a given $r<0$ close to zero.

Analytical conditions for stability can be found in Stépán [70] and Hassard [42]. These conditions are deduced by using the argument principle of complex analysis, and they give a practical method for determining stability. In recent years, numerical methods have been developed to compute approximations to the right-most (stability-determining) characteristic roots of (4), by using a discretization either of the solution operator of (3) or of the infinitesimal generator of the semi-group of the solution operator of (3). The solution operator $\mathcal{S}(t)$ of the linearized equation (3) maps an initial function segment onto the solution segment at time $t$, i.e.,

$$
\mathcal{S}(t) y(\cdot)(\theta)=y(t+\theta), \quad-\tau \leq \theta \leq 0, t \geq 0 .
$$

This operator has eigenvalues $\mu$, which are related to the characteristic roots via the equation $\mu=e^{\lambda t}$ [63]. To determine the stability, we are interested in the dominant eigenvalues that, if $t$ is large, are well separated. This can be an advantage for the eigenvalue computation, but the time integration itself may be costly. In Sec. 1.1 we describe a reliable way to compute the dominant eigenvalues of $\mathcal{S}(h)$ where $h$ is the time step of a linear multistep (LMS) method.

Since $\mathcal{S}(t)$ is a strongly continuous semi-group [36, 38], one can define the corresponding infinitesimal generator $\mathcal{A}$ by

$$
\mathcal{A} y=\lim _{t \rightarrow 0+} \frac{\mathcal{S}(t) y-y}{t} .
$$

For (3) the infinitesimal generator becomes

$$
\begin{aligned}
& \mathcal{A} y(\theta)=y^{\prime}(\theta), y \in \mathcal{D}(\mathcal{A}) \\
& \mathcal{D}(\mathcal{A})=\left\{y \in C: y^{\prime} \in C \quad \text { and } \quad y^{\prime}(0)=\sum_{j=0}^{m} A_{j} y\left(-\tau_{j}\right)\right\}
\end{aligned}
$$

Both operators can be discretised by spectral discretizations or time integration methods; this always leads to a representation by some matrix. Eigenvalues of this matrix yields approximations to the right-most characteristic roots. Hence, for computational efficiency it is important that the size of 
the resulting matrix eigenvalue problem is small, or at least that the stability determining eigenvalues can be computed efficiently. iterative method, such as subspace iteration. Accurate characteristic roots can be found by using Newton iterations on the characteristic equation

$$
\begin{aligned}
& \left(\lambda I-A_{0}-\sum_{j=1}^{m} A_{j} \mathrm{e}^{-\lambda \tau_{j}}\right) v=0, \\
& v_{0}^{T} v=1
\end{aligned}
$$

where $v \in \mathbb{R}^{n}$ and $v_{0} \in \mathbb{R}^{n}$, to obtain accurate characteristic roots $\lambda$ (and the corresponding eigenfunctions $v e^{\lambda t}$ ). The difference between the approximate and the corrected roots gives an indication of the accuracy of the approximations.

Below we describe how the characteristic roots can be computed via an approximation of the solution operator by time integration, which is the method that is implemented in DDE-BIfTOOL. We also briefly comment on other approaches.

\subsection{Approximation of the solution operator by a time integrator}

A natural way to approximate the solution operator is to write the numerical time integration of the linearized equation as a matrix equation. Engelborghs et al. [27] have proposed and analysed the use of a linear multistep method with constant steplength $h$ to approximate the solution operator $\mathcal{S}(h)$. The delay interval $[-\tau, 0]$ (slightly extended to the left and the right; see below) is discretized by using an equidistant mesh with mesh spacing $h$, and a solution is represented by a discrete set of points $y_{i}:=y\left(t_{i}\right)$ with $t_{i}=i h$. A $k$-step LMS method with steplength $h$ to compute $y_{k}$ can be written as

$$
\sum_{i=0}^{k} \alpha_{i} y_{i}=h \sum_{i=0}^{k} \beta_{i}\left(A_{0} y_{i}+\sum_{j=1}^{m} A_{j} \tilde{y}\left(t_{i}-\tau_{j}\right)\right),
$$

where $\alpha_{i}$ and $\beta_{i}$ are parameters and where (in case $t_{i}-\tau_{j}$ does not coincide with a mesh point) the approximations $\tilde{y}\left(t_{i}-\tau_{j}\right)$ are obtained by polynomial interpolation with $s_{-}$and $s_{+}$points to the left and the right, respectively.

The discretization of the solution operator is the (linear) map between $\left[y_{L_{m i n}}, \ldots, y_{k-1}\right]^{T}$ and $\left[y_{L_{m i n}+1}, \ldots, y_{k}\right]^{T}$ where $L_{m i n}=-s_{-}-\lceil\tau / h\rceil$ and where the mapping is defined by (9) for $y_{k}$ and by a shift for all variables other than $y_{k}$. This map is represented by an $N \times N$ matrix, where

$$
N=n\left(-L_{\min }+k\right) \approx n \tau / h .
$$

Since the time step $h$ is small, the eigenvalues $\mu$ of this matrix are not well separated (most eigenvalues lie close to the unit circle). They can be computed by e.g. the QR method, with a computational cost of the order $N^{3} \approx n^{3}(\tau / h)^{3}$, and so approximations to the characteristic roots can be derived. 
To guarantee the reliability of the stability computation, the steplength $h$ in the LMS method (9) should be chosen such that all characteristic roots $\lambda$ with $\operatorname{Re}(e(\lambda)) \geqslant r(r<0)$ are approximated accurately. Procedures for such a safe choice of $h$ are described in [27,79] and implemented in DDE-BIfTool. They are based on theoretical properties of

(a) the relation between the stability properties of the solution of the linearized equation (3) and the stability of the discretized equation (9);

(b) an a-priori estimate of the region in the complex plane that includes all characteristic roots $\lambda$ with $\operatorname{Re}(e(\lambda)) \geqslant r$.

Note that the solution operator can also be discretized by using a RungeKutta time integrator [10].

\subsection{Other approaches}

Breda et al. [11] have developed numerical methods to determine the stability of solutions based on a discretization of the infinitesimal generator. By discretizing the derivative in (7) with a Runge-Kutta or a LMS method, a matrix approximation of $\mathcal{A}$ is obtained. The resulting eigenvalue problem is large and sparse, as in the case when the solution operator is discretized by a time integration method. Breda et al. [12] also proposed a pseudo-spectral discretization of the infinitesimal generator. In this approach, an eigenfunction of the infinitesimal generator $v e^{\lambda t}, t \in[-\tau, 0]$, is approximated by a polynomial $P(t)$ of degree $p$. Collocation for the eigenvalue problem for the infinitisimal generator leads to an equation of the form

$$
P^{\prime}\left(t_{i}\right)=\lambda P\left(t_{i}\right)
$$

where the collocation points $t_{i}, i=1 \ldots p$ are chosen as the shifted and scaled roots of an (orthogonal) polynomial of degree $p$. These equations are augmented with

$$
A_{0} P(0)+\sum_{j=1}^{m} A_{j} P\left(\tau_{j}\right)=\lambda P(0),
$$

which introduces the system-dependent information. The resulting matrix eigenvalue problem has size $n(p+1)$. The first $n p$ rows are the Kronecker product of a dense $p \times(p+1)$ matrix and the identity matrix. The last block row consists of a linear combination of the matrices $A_{j}, j=0, \ldots, m$ and the identity. The matrix is full but can be of much smaller size than in the previous case, due to the 'spectral accuracy' convergence, as is shown in the detailed analysis presented in [12].

A pseudo-spectral discretization of the solution operator is proposed in $[81,10]$. Here a polynomial approximation $P(t)$ of an eigenfunction, defined on the interval $[-\tau, h]$ has to satisfy $p$ collocation conditions of the form

$$
P\left(t_{i}+h\right)=\mu P\left(t_{i}\right)
$$


where $\mu=e^{\lambda h}$. These equations are augmented with a condition obtained from integrating the linearized equations over an time interval $h$. When high accuracy is required, a pseudo-spectral discretization will lead to a more efficient procedure than when a time integration discretization is used, but numerical experiments indicate that for low accuracy requirements both approaches are competitive [81].

However, for the pseudo-spectral approaches no strategy is known that guarantees a priori that all characteristic roots with real part larger than $r$ are computed accurately, as is the case with the discretization of the solution operator with a LMS method.

\section{Periodic solutions}

A periodic solution $x^{\star}(t)$ of an autonomous system of the form (1) satisfies

$$
x^{\star}(t+T)=x^{\star}(t), \forall t,
$$

where $T$ is the period. An extensive literature exists on the existence, stability and parameter dependence of periodic solutions; see, e.g., [38, §XI.1-2]. These results are essentially analytical in nature and the corresponding methods have different rigorous restrictions and cannot be applied to general nonlinear systems with several delays.

Because of the dependence on the past, periodicity of $x(t)$ at one moment in time, $x(t)=x(t+T)$ for some $t$, does not imply periodicity for the whole solution. Instead, a complete function segment of length $\tau$ has to be repeated. Consequently, a periodic solution to (1) can be found as the solution of the following two-point boundary value problem (BVP),

$$
\left\{\begin{array}{l}
x^{\prime}(t)=f\left(x(t), x\left(t-\tau_{1}\right), \ldots, x\left(t-\tau_{m}\right), \eta\right), t \in[0, T] \\
x_{0}=x_{T} \\
p(x, T)=0
\end{array}\right.
$$

where $x_{0}$ and $x_{T}$ are function segments on $[-\tau, 0]$ and $[-\tau+T, T]$, respectively, the period $T$ is an unknown parameter. Furthermore, $p$ represents a phase condition that is needed to remove translational invariance. A well-known example is the classical integral phase condition [20]

$$
\int_{0}^{1} \dot{u}^{(0)}(s)\left(u^{(0)}(s)-u(s)\right) \mathrm{d} s=0,
$$

where $u^{(0)}$ is a reference solution; see also Chapter 1 .

Stable periodic solutions of a DDE can be found by numerical time integration; the convergence of the integration depends on the stability properties of the periodic solution [44]. However, both stable and unstable solutions can be computed by solving the above boundary value problem by either collocation or by approach. Here we only consider collocation methods. 


\subsection{Collocation}

In collocation a periodic solution is computed by using a discrete representation that satisfies the differential equation at a set of collocation points on $[0, T]$. Doedel and Leung [21] have computed periodic solutions of DDEs using collocation based on a truncated Fourier series; see also [13] for a similar approach. This Fourier approach has the advantage that periodicity is automatically fulfilled. However, steep gradients in a solution pose problems and it is not possible to determine the solution stability.

Collocation based on piecewise polynomial representations is used in Auto [17] and Content [50] to compute periodic solutions for systems of ordinary differential equations; see also Chapters 1and 2. We now discuss how piecewisepolynomial collocation can be used for DDEs. We first rescale time by a factor $1 / T$ such that the period is one in the transformed system

$$
\left\{\begin{array}{l}
x^{\prime}(t)=T f\left(x(t), x\left(t-\tau_{1} / T\right), \ldots, x\left(t-\tau_{m} / T\right), \eta\right), \quad \text { for } t \in[0,1], \\
x(\theta+1)-x(\theta)=0, \quad \text { for } \theta \in[-\tau / T, 0], \\
p(x, T)=0 .
\end{array}\right.
$$

A mesh with $L+1$ mesh points $\left\{0=t_{0}<t_{1}<\ldots<t_{L}=1\right\}$ is specified. This mesh is periodically extended to the left with $\ell$ points to obtain a mesh on $[-\tau / T, 1]$ with $\ell+L$ intervals. In each interval an approximating polynomial of degree $d$ is described in terms of the function values at the representation points (using Lagrange polynomials as basis). These function values are determined by requiring that the approximating collocation solution fulfills the (time-scaled) differential equations exactly at the collocation points. In each interval, the collocation points are typically chosen as the (scaled and shifted) roots of a $d$-th degree orthogonal polynomial.

The approximating polynomial of degree $d$ on each interval $\left[t_{i}, t_{i+1}\right], i=$ $-\ell, \ldots, L-1$, can be written as

$$
u(t)=\sum_{j=0}^{d} u\left(t_{i+\frac{j}{d}}\right) P_{i, j}(t), t \in\left[t_{i}, t_{i+1}\right],
$$

where $P_{i, j}(t)$ are the Lagrange polynomials through the representation points

$$
t_{i+\frac{j}{d}}=t_{i}+\frac{j}{d}\left(t_{i+1}-t_{i}\right), \quad j=0, \ldots, d .
$$

Because polynomials on adjacent intervals share the value at the common mesh point, this representation is automatically continuous (however, it is not continuously differentiable at the mesh points).

The approximation $u(t)$ is completely determined by the coefficients

$$
u_{i+\frac{j}{d}}:=u\left(t_{i+\frac{j}{d}}\right), i=-\ell, \ldots, L-1, j=0, \ldots, d-1 \text { and } u_{L}:=u\left(t_{L}\right) .
$$

We define the starting vector $u_{s}$ and the final vector $u_{f}$, both of length $N=$ $n(\ell d+1)$, as 


$$
u_{s}:=\left[u_{-\ell}, \ldots, u_{i+\frac{j}{d}}, \ldots, u_{0}\right]^{T}, \quad u_{f}:=\left[u_{L-\ell}, \ldots, u_{i+\frac{j}{d}}, \ldots, u_{L}\right]^{T} .
$$

The collocation points are obtained as

$$
c_{i, j}=t_{i}+c_{j}\left(t_{i+1}-t_{i}\right), i=0, \ldots, L-1, j=1, \ldots, d
$$

from a set of collocation parameters $c_{j}, j=1, \ldots, d$, e.g., the shifted and scaled roots of the $d$-th degree Gauss-Legendre polynomial.

A periodic solution for a fixed value of the parameters $\eta$ is found as the solution of the following $(n((\ell+L) d+1)+1) \times(n(\ell+L) d+1)+1)$-dimensional (nonlinear) system in terms of the unknowns (17) and $T$,

$$
\left\{\begin{array}{l}
\left.\dot{u}\left(c_{i, j}\right)=T f\left(u\left(c_{i, j}\right), u\left(c_{i, j}-\frac{\tau_{1}}{T}\right), \ldots, u\left(c_{i, j}-\frac{\tau_{m}}{T}\right)\right), \eta\right)=0 \\
i=0, \ldots, L-1, j=1, \ldots, d
\end{array}\right)
$$

Here, $p$ again represents a phase condition such as (14).

The collocation solution fulfils the time-scaled differential equation exactly at the collocation points. If $c_{i, j}$ coincides with $t_{i}$ then the right derivative is taken in (19), if it coincides with $t_{i+1}$ then the left derivative is taken. Taking into account the periodicity conditions, one can reduce system (19) to the following nonlinear system in the unknowns $u=\left[u_{0}, \ldots, u_{L}\right]^{T}$ and $T$,

$$
\left\{\begin{array}{l}
\dot{u}\left(c_{i, j}\right)=T f\left(u\left(c_{i, j}\right), u\left(\left(c_{i, j}-\frac{\tau_{1}}{T}\right) \bmod 1\right), \ldots, u\left(\left(c_{i, j}-\frac{\tau_{m}}{T}\right) \bmod 1\right), \eta\right)=0, \\
\quad i=0, \ldots, L-1, j=1, \ldots, d \\
\begin{array}{l}
u_{0}=u_{L}, \\
p(u)=0
\end{array}
\end{array}\right.
$$

Hence, the dimension of the system and the number of unknowns is reduced to $(n(L d+1)+1)$.

When using Newton's method to solve (20), the matrix of the linear system to be solved in each iteration is sparse and has a particular structure, as is shown in Fig. 1. The matrix consists of a (large) $n L d \times n(L d+1)$ matrix filled with two (circular) bands, bordered by one column and $n+1$ rows. The extra column contains derivatives with respect to the period; $n$ extra rows contain the periodicity condition, and one extra row is due to the phase condition (14). The diagonal band is itself a concatenation of $n d \times n(d+1)$ blocks. The off-diagonal bands are a consequence of the delay terms. When the mesh is equispaced then the off-diagonal band lies at a fixed distance from the diagonal band as is illustrated in Fig. 1(a). This is no longer the case when the mesh is non-equispaced; see Fig. 1(b).

In the case of collocation for ODEs, the matrix of the linear system has a band structure with a band size proportional to $n$ and $d$ but independent of the number of mesh intervals $L$; see also Chapter 1 . Hence the system can be solved efficiently by a direct band solver. For delay differential equations this 

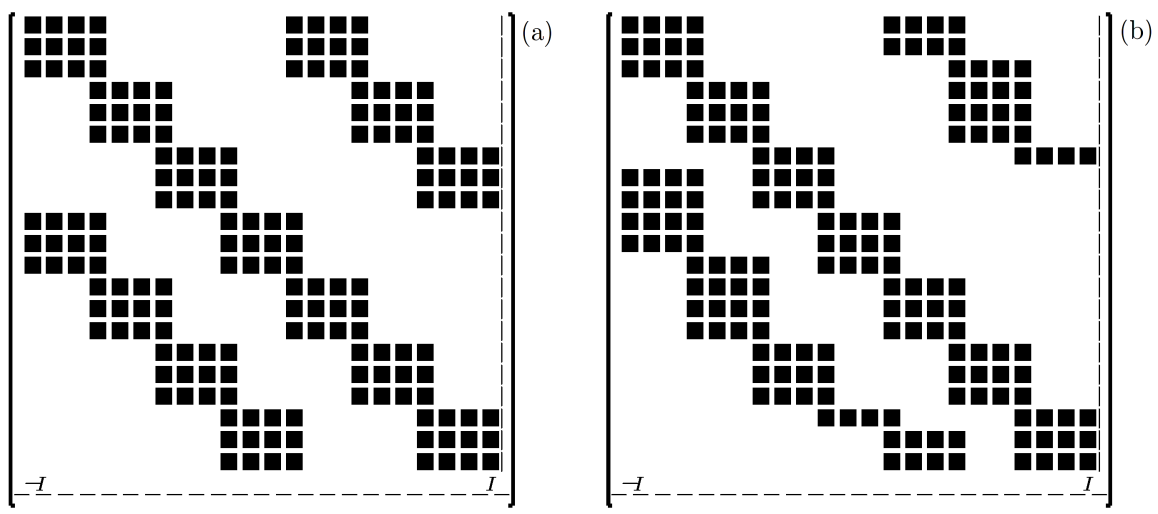

Fig. 1. Structure of the matrix arising in the Newton iteration to solve (20) for one delay that is smaller than the period $T$; shown is the case $L=7$ and collocation polynomials of degree $d=3$. Panel (a) is for an equispaced mesh and panel (b) for a non-equispaced mesh; each black box represents an $n \times n$ block.

is not possible. Indeed, the structure of the matrix, described above, cannot easily be exploited when using a direct solver, especially in case of several delays and/or a non-equispaced mesh. However, for moderate values of $d, n$ and $L$ the linear system (20) can still be solved with a direct method. The efficiency can be increased by using a chord-Newton method, in which case the Jacobian is not recomputed (and factored) in every iteration but remains fixed during a number of iterations. In Sec. 2.3 we describe an efficient iterative procedure to solve (20).

Furthermore, an adaptive (non-equispaced) mesh can be used to decrease the required number of intervals $L$ for difficult solutions (with steep gradients). For the latter, the interval size $h_{i}=t_{i+1}-t_{i}$ is adapted to an approximation of the $(d+1)$-th derivative of the solution (obtained from the computed solution; see $[1,24])$.

Engelborghs and Doedel [23] have proven that the convergence rate of the maximal continuous error $E=\max _{t \in[0,1]}\left\|u(t)-u^{\star}(t)\right\|$ is $\mathcal{O}\left(h^{d}\right)$ in general and $\mathcal{O}\left(h^{d+1}\right)$ for Gauss-Legendre collocation points on equispaced and nonequispaced meshes with $h=\max _{i} h_{i}$. Special convergence rates at the mesh points (so-called superconvergence) that feature for ordinary differential equations, are, in general, lost for DDEs.

Note that, in the case of a non-autonomous (or forced) system, the collocation method is essentially the same as in (20), except that the phase condition is not needed, since the phase of the solution is determined by the phase of the forcing. 


\subsection{Monodromy operator and Floquet multipliers}

The stability of a periodic solution is determined by the Floquet multipliers, which are the eigenvalues of the monodromy operator. In the case of autonomous equations there is always a trivial multiplier +1 , which stems from the fact that the associated linearized equation is always solved by the time derivative of the solution itself. According to Floquet theory, a periodic solution is asymptotically stable if all the multipliers - not counting the trivial one lie strictly inside the complex unit disk. The main focus of this subsection is the computation of the monodromy operator using the previously described collocation method.

Benote by $x^{\star}(t)$ a $T$-periodic solution of (1). As in the previous sections we rescale time by $1 / T$. The linearized equation about this periodic solution in rescaled time is

$$
\frac{d}{d t} y(t)=T\left(A_{0}(t) y(t)+\sum_{j=1}^{m} A_{j}(t) y(t-\tau)\right),
$$

where $A_{j}(t)$ denotes the partial derivative of $f$ with respect to its $(j+1)$-th argument, evaluated at $x^{\star}(T t)$. Also let $U(t, s)$ be the fundamental solution operator of (21), which is defined as

$$
\left(U(t, s) \phi_{s}\right)(\theta)=y(t+\theta), \quad \theta \in[-\tau / T, 0],
$$

where $\phi_{s}$ is an initial function and $y$ is the corresponding solution of (21). The monodromy operator is defined as

$$
\mathcal{M}=U(1,0),
$$

that is

$$
\begin{aligned}
\mathcal{M}: C\left([-\tau / T, 0] ; \mathbb{R}^{n}\right) & \rightarrow C\left([-\tau / T, 0] ; \mathbb{R}^{n}\right), \\
\phi & \mapsto y_{1},
\end{aligned}
$$

where $\phi$ is the initial function and $y_{1}$ is the solution segment $y_{1}(\theta)=y(1+\theta)$. The discretized version of $\mathcal{M}$ is $\mathcal{M}_{d}: u_{s} \rightarrow u_{f}$ and its matrix representation can be obtained by solving (21) with a collocation method similar to (19). This method is used in DDE-BIFTool [26].

However, when the maximal delay is larger than the period, $u_{s}$ and $u_{f}$ overlap; computation of $\mathcal{M}_{d}$ can be improved by exploiting this property. For the sake of generality we use the Riesz representation theorem and write (21) in the form

$$
\frac{\mathrm{d} y(t)}{\mathrm{d} t}=T \int_{0}^{\tau / T} \mathrm{~d}_{\theta} \zeta(T \theta, t) y(t-\theta),
$$

where $\zeta$ is a matrix valued function of bounded variation that, with (21), can be written as 


$$
\zeta(T \theta, t)=\left\{\begin{array}{ccc}
0 & \text { if } & \theta \leq 0 \\
A_{0}(t) & \text { if } 0<\theta<\tau_{1} \\
\vdots & & \vdots \\
A_{0}(t)+\sum_{j=1}^{m} A_{j}(t) & \text { if } & \tau \leq \theta
\end{array} .\right.
$$

Notice that (22) implicitly depends on the initial function. It can be written explicitly as

$$
\frac{\mathrm{d} y(t)}{\mathrm{d} t}-T \int_{0}^{t} \mathrm{~d}_{\theta} \zeta(T \theta, t) y(t-\theta)-T \int_{t}^{\tau / T} \mathrm{~d}_{\theta} \zeta(T \theta, t) \phi(t-\theta)=0 .
$$

We introduce $K=\lceil\tau / T\rceil$ solution segments of $y(t)$ and $\phi$ as

$$
\begin{aligned}
& y_{1}(t)=y(2-K+t), y_{2}(t)=y(3-K+t), \ldots, y_{K}(t)=y(1+t) \\
& \phi_{1}(t)=\phi(1-K+t), \phi_{2}(t)=\phi(2-K+t), \ldots, \phi_{K}(t)=\phi(t)
\end{aligned} \quad, t \in[-1,0],
$$

such that $\phi_{i}, y_{i} \in \mathcal{X}:=C\left([-1,0] ; \mathbb{R}^{n}\right)$, and we also define operators on $\mathcal{X}$ as obtained from from $(23)$ as

$$
\begin{gathered}
(\mathcal{A} \phi)(\theta)=\frac{\mathrm{d} \phi(\theta)}{\mathrm{d} t}-T \int_{0}^{1+\theta} \mathrm{d}_{\gamma} \zeta(T \gamma, \theta) \phi(\theta-\gamma), \quad D(\mathcal{A})=C^{1}\left([-1,0], \mathbb{R}^{n}\right) \\
\left(\mathcal{B}_{i} \phi\right)(\theta)=T \int_{i+\theta}^{i+1+\theta} \mathrm{d}_{\gamma} \zeta(T \gamma, \theta) \phi(i+\theta-\gamma), \quad 1 \leqslant i \leqslant N
\end{gathered}
$$

It is clear that $y_{K}$ is the only unknown, because all the other $y_{i}$ can be found from the initial conditions as $y_{i}=\phi_{i+1}$. Hence, the only equation that has to be solved is

$$
\mathcal{A} y_{K}-\sum_{i=1}^{K} \mathcal{B}_{i} \phi_{i}=0, \quad y_{K}(-1)=\phi_{K}(0) .
$$

In order to eliminate the explicit boundary condition we introduce extended operators on $\hat{\mathcal{X}}=\left\{(\varphi, c) \in \mathcal{X} \times \mathbb{R}^{n}: c=\varphi(0)\right\}$ in the form of

$$
\hat{\mathcal{A}}=\left(\begin{array}{ll}
\mathcal{A} & 0 \\
\mathcal{L} & 0
\end{array}\right), \quad \hat{B}_{i}=\left(\begin{array}{cc}
\mathcal{B}_{i} & 0 \\
0 & 0
\end{array}\right) \text { for } i<N \quad \text { and } \quad \hat{B}_{N}=\left(\begin{array}{cc}
\mathcal{B}_{N} & 0 \\
0 & I
\end{array}\right),
$$

where $\mathcal{L} \varphi=\varphi(-1)$. The extended monodromy operator is defined on $X=$ $C\left([-N, 0] ; \mathbb{R}^{n}\right)$; this space is isomorphic to the further extended

$\tilde{X}=\left\{\left(\left(\phi_{1}, c_{1}\right), \ldots,\left(\phi_{N}, c_{N}\right)\right) \in \hat{\mathcal{X}}^{N}: \phi_{k}(0)=c_{k}=\phi_{k+1}(-1), 1 \leqslant k<N\right\}$.

In order to obtain stability results it is sufficient to construct the monodromy operator on $\tilde{X}$, which becomes 


$$
\tilde{\mathcal{M}}=\left(\begin{array}{cccc}
0 & \hat{I} & \cdots & 0 \\
0 & 0 & \ddots & 0 \\
\vdots & \vdots & & \vdots \\
0 & 0 & & \hat{I} \\
\hat{\mathcal{A}}^{-1} \hat{\mathcal{B}}_{1} & \hat{\mathcal{A}}^{-1} \hat{\mathcal{B}}_{2} & \cdots & \hat{\mathcal{A}}^{-1} \hat{\mathcal{B}}_{N}
\end{array}\right)
$$

Because of the identity matrices above the diagonal, the operator $\tilde{\mathcal{M}}$ is not compact, but its powers $\tilde{\mathcal{M}}^{k}, k \geqslant K$ are compact.

The operator $\tilde{\mathcal{M}}$ can be computed by collocation and by inverting the resulting discretized $\hat{\mathcal{A}}$ operator. In PDDE-CONT the spectrum of $\tilde{\mathcal{M}}$ is computed with the iterative Arnoldi-Lanczos method [64], which is implemented in the ARPACK software package. Note that in this iterative process, when the discretized operator is multiplied by a vector, only one solution step with $\hat{\mathcal{A}}$ is necessary.

Despite the differences, using either $\mathcal{M}$ or $\tilde{\mathcal{M}}$ gives the same accuracy of the multiplier calculation [53]. In particular, it was shown in [53] that the computations of the multipliers and of the periodic solution itself have the same accuracy. The exception is the computations of the trivial multiplier +1 , which was found to be more accurate. Hence, inferring the accuracy of the periodic solution from the accuracy of the trivial multiplier can be deceiving.

\subsection{Collocation-Newton-Picard}

Verheyden and Lust [78] have developed an iterative procedure to solve the linear system arising in Newton's method applied to system (19). Consider the unknowns $u_{i+j / d}:=u\left(t_{i+j / d}\right)$ defined in (17). Recall the definition of the starting vector $u_{s}$ and the final vector $u_{f}$ given in (18)

$$
u_{s}:=\left[u_{-\ell}, \ldots, u_{i+\frac{j}{d}}, \ldots, u_{0}\right]^{T}, \quad u_{f}:=\left[u_{L-\ell}, \ldots, u_{i+\frac{j}{d}}, \ldots, u_{L}\right]^{T},
$$

and define the trajectory vector as

$$
u_{t}:=\left[u_{\frac{1}{d}}, \ldots, u_{i+\frac{j}{d}} \ldots, u_{L}\right]^{T},
$$

where $u_{s}$ and $u_{f}$ are of length $N=n(\ell d+1)$ and $u_{t}$ is of length $\hat{N}=n L d(\ell$ and $L$ denote the number of mesh points in $[-\tau / T, 0]$ and $(0,1]$, respectively). Note that $u_{f}$ consists of the last $n(\ell d+1)$ components of $u_{t}$.

The linearization of (19) has the following form

$$
\begin{aligned}
-B \Delta u_{s}+A \Delta u_{t}+r_{1, T} \Delta T & =-r_{1} \\
\Delta-u_{s}+\Delta u_{f} & =-r_{2} \\
\alpha_{s} \Delta u_{s}+\alpha_{t} \Delta-u_{t}+\alpha_{T} \Delta T & =-\alpha
\end{aligned}
$$

where $r_{1}, r_{2}$ and $\alpha$ denote the residuals of system (19) and $-B, A$ and $r_{1, T}$ denote the partial derivatives of the collocation conditions with respect to $u_{s}$, 

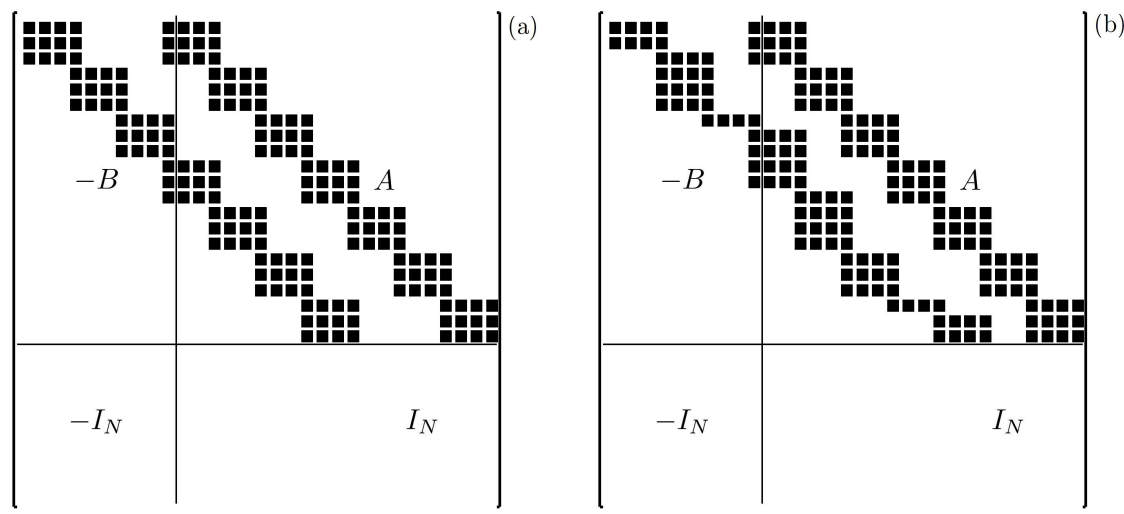

Fig. 2. Typical structure of the linearized collocation system for one delay that is smaller than the period $T$; shown is the case of $\ell+L=3+7$ mesh intervals and collocation polynomials of degree $d=3$ (the bordering row and column are omitted). Panel (a) is for an equispaced mesh and panel (b) for a non-equispaced mesh; each black box represents an $n \times n$ block.

$u_{t}$ and $T$. A typical structure for the matrix of the linearized system in the case of one time delay that is smaller than the period $T$ is shown in Fig. 2. Panel (a) is for an equispaced mesh, while panel (b) a non-equispaced mesh is used with $L=7$ mesh intervals; the extended mesh contains $\ell=3$ additional mesh intervals and the piecewise polynomials have degree $d=3$.

The linear system can be manipulated and condensed to the form

$$
\left[\begin{array}{cc}
M-I & b_{c} \\
\beta_{s} & \beta_{T}
\end{array}\right]\left[\begin{array}{c}
\Delta u_{s} \\
\Delta T
\end{array}\right]=-\left[\begin{array}{l}
-r_{c} \\
-\alpha_{c}
\end{array}\right]
$$

Here $M$ is the discretization of the monodromy operator, which can be derived from $M_{t}=A^{-1} B$. Afterwards, $\Delta u_{t}$ can be computed from (27). This manipulation is based on the correspondence between the linearization of the collocation scheme and the discretization of the linearized boundary value problem. The condensation is similar to the condensation used in AUTO; see Chapter 1. The monodromy matrix (28) is well suited for the Newton-Picard method [52], which leads to a substantial reduction in the computational cost, especially when only a few Floquet multipliers are larger in modulus than a certain threshold $\rho$, e.g., $\rho=0.5[78]$.

\section{Defining systems for codimension-one bifurcations of periodic solutions}

Periodic solutions of autonomous DDEs can undergo three generic codimensionone bifurcations. First,the monodromy operator may have an algebraically 
double +1 eigenvalue, which corresponds to a limit point (or fold, or saddlenode) bifurcations where the solution ceases to exist. Secondl, there may be a single -1 multiplier, which gives a period-doubling bifurcation. Third, if two critical complex conjugate multipliers lie on the unit circle of the complex plane, then there is a Neimark-Sacker or torus bifurcation. In this case an invariant torus bifurcates from the periodic solution.

Continuing the bifurcations of periodic solutions in DDEs does not differ substantially from the case of ODEs. In order to compute bifurcations one has to include additional equations to (20), which are satisfied by a periodic solution if and only if the monodromy operator has a certain kind of singularity. In the period-doubling and the Neimark-Sacker cases, the simplest procedure to construct such a determining system is to require that the monodromy operator has a singular vector. Short algebraic transformations of (24) reveals that these bifurcations occur if

$$
\begin{aligned}
\left(\hat{\mathcal{A}}-\sum_{j=1}^{N} \sigma^{j} \hat{\mathcal{B}}_{j}\right) v & =0, \\
v^{\star} v & =1,
\end{aligned}
$$

has a unique solution $v$ with the inverse characteristic multiplier $\sigma=\mu^{-1} \neq 1$ on the unit circle. Because of the appearance of higher powers of $\sigma$, this equation is different from the ODE case if the delay is larger than the period. Adding (29) to the defining system of the periodic solution (20) doubles the size of the problem. The size of (29) can be reduced to $n+1$ by using characteristic matrices that are equivalent to the operator in (29) [75]. However, the smallest possible addition would consist of only one additional scalar equation to (20) without introducing new variables. This can be achieved by using the bordering theorem [8], which states that the bordered operator

$$
\left(\begin{array}{cc}
D & \beta \\
\alpha^{\star} & \delta
\end{array}\right)=\left(\begin{array}{ll}
A & b \\
c^{\star} & 0
\end{array}\right)^{-1},
$$

exists if both $A$ and $A^{*}$ have one-dimensional kernels and $b \notin \operatorname{ker} A^{*}, c \notin \operatorname{ker} A$ or $A$ is bijective and $c^{\star} A^{-1} b \neq 0$. Moreover, $\delta$ can be used as a test functional of the singularity, because it is zero if and only if $A$ is singular. In order to obtain $\delta$ it is sufficient to solve the equation

$$
\left(\begin{array}{ll}
A & b \\
c^{\star} & 0
\end{array}\right)\left(\begin{array}{l}
\beta \\
\delta
\end{array}\right)=\left(\begin{array}{l}
0 \\
1
\end{array}\right) .
$$

Hence, using a discretized version of $\hat{\mathcal{A}}-\sum_{j=1}^{N} \sigma^{j} \hat{\mathcal{B}}_{j}$ for the operator $A$ in (30) with appropriate choices of $b$ and $c^{\star}$ in the period-doubling and NeimarkSacker case, the equation $\delta\left(x^{*}, \eta\right)=0$ determines the bifurcation point. In a continuation context the resulting $\beta$ can be re-used as the value of $c$ in the next continuation step. Similarly, by solving the adjoint equation 


$$
\left(\begin{array}{ll}
A^{\star} & c \\
b^{\star} & 0
\end{array}\right)\left(\begin{array}{l}
\alpha \\
\delta
\end{array}\right)=\left(\begin{array}{l}
0 \\
1
\end{array}\right)
$$

the resulting $\alpha$ can be the new value of $b$ in the next continuation step.

In the case of the fold bifurcation in an autonomous system (1), because of the algebraically double +1 multiplier, the operator $A$ has to be

$$
A_{L P}=\left(\begin{array}{cc}
\hat{\mathcal{A}}-\sum_{j=1}^{N} \sigma^{j} \hat{\mathcal{B}}_{j} & \phi_{0} \\
\operatorname{Int}_{\psi_{0}} & 0
\end{array}\right),
$$

where

$$
\begin{gathered}
\psi_{0}=f\left(x(t), x\left(t-\tau_{1}\right), x\left(t-\tau_{2}\right), \ldots, x\left(t-\tau_{m}\right), \eta\right), \\
\phi_{0}=-\sum_{j=1}^{N} j \hat{\mathcal{B}}_{j} \psi_{0}
\end{gathered}
$$

and

$$
\operatorname{Int}_{\psi_{0}} \phi=\int_{-1}^{0} \psi_{0}(\theta) \phi(\theta) d \theta
$$

Note that $A_{L P}$ is different from what one would expect by analogy with ODEs; see [19]. Here, $\phi_{0}$ is obtained by computing the Jordan chain of $\hat{\mathcal{A}}-\sum_{j=1}^{N} \sigma^{j} \hat{\mathcal{B}}_{j}$; see, e.g., [46]. The regularity of $\delta$ obtained from $A_{L P}$ at the bifurcation point can be proven either by using the equivalence with characteristic matrices [75] or by standard techniques [19].

\section{Connecting orbits}

A solution $x^{\star}(t)$ of (1) at some fixed value of the parameter $\eta$ is called a connecting orbit if the limits

$$
\lim _{t \rightarrow-\infty} x^{\star}(t)=x^{-} \quad \text { and } \quad \lim _{t \rightarrow+\infty} x^{\star}(t)=x^{+}
$$

exist, where $x^{-}$and $x^{+}$are steady states of (1). We call the orbit homoclinic when $x^{-}=x^{+}$, and heteroclinic otherwise. Orbits of this type exist, for instance, in lasers models with optical feedback, which are discussed in Sec. 8.1; see also [34]. They also appear naturally when looking for traveling waves in delay partial differential equations [66].

A defining condition for a connecting orbit is that it is contained in both the stable manifold of $x^{+}$and the unstable manifold of $x^{-}$. A classical approach in the ODE case is to approximate this condition by truncating the time domain to an interval of length $T_{c}$ and to apply (so-called) projection boundary conditions [7]: one end point of the connecting orbit is required to lie in the unstable eigenspace of $x^{-}$and the other end point in the stable 
eigenspace of $x^{+}$. The projection boundary conditions, therefore, replace the stable and unstable manifolds by their linear approximations near the steady states.

Here, the boundary conditions need to be written in terms of solution segments. Furthermore, $x^{+}$has infinitely many eigenvalues with negative real parts (see Sec. 1) and so it is impossible to write the final function segment as a linear combination of all stable eigenfunctions. Instead, it is required that the end function segment is in the orthogonal complement of all unstable left eigenfunctions. We will assume for notational convenience that (1) only contains one delay $\tau$; however, the method is implemented in DDE-BIFTOOL for the general case of $m$ fixed delays.

The condition for the initial function segment $x_{0}(\theta)$ can be written as

$$
x_{0}(\theta)=x^{-}+\varepsilon \sum_{k=1}^{s^{-}} \alpha_{k} v_{k}^{-} e^{\lambda_{k}^{-} \theta} \quad\left(\sum\left|\alpha_{k}\right|^{2}=1\right),
$$

where $s^{-}$is the number of unstable eigenvalues $\lambda^{-}$, with corresponding eigenvectors $v^{-}$. The $\alpha_{k}$ are unknown coefficients, and $\varepsilon$ is a measure for the desired accuracy. An extra condition is added to ensure continuity at $\theta=0$. Since we cannot write the end conditions for the final function segment in a similar way, a special bilinear form [36] is used to express the fact that the final function segment is in the complement of the unstable eigenspace of $x^{+}$. This leads to the $s^{+}$extra conditions of the form

$$
w_{k}^{+^{*}}\left(x\left(T_{c}\right)-x^{+}\right)+\int_{-\tau}^{0} w_{k}^{+^{*}} e^{-\lambda_{k}^{+}(\theta+\tau)} A_{1}\left(x^{+}, \eta\right)\left(x\left(T_{c}+\theta\right)-x^{+}\right) d \theta=0,
$$

where $k=1, \ldots, s^{+}$. Here $s^{+}$is the number of unstable eigenvalues of $x^{+}, w_{k}^{+}$ are the left eigenvectors corresponding to the eigenvalues $\lambda_{k}^{+}$, and the matrix $A_{1}$ is defined as in (3). While this integral condition works well in practice, one slight drawback is that it does not control the distance of the end function segment to the steady state.

As for periodic solutions, connecting orbits arise in one-parameter families and any time-translate is also a connecting orbit. Therefore, a phase condition such as (14) needs to be added to select one of these orbits.

For the case of a one-parameter family of connecting orbits a number of free parameters are required to obtain a generically isolated solution. One has to solve (1) together with the steady-state equations (2) for $x^{-}$and $x^{+}$ and characteristic equations of the form (8) for $\lambda_{k}^{-}$and $v_{k}^{-}$and $\lambda_{k}^{+}$and $w_{k}^{+}$, i.e., a system of $n$ differential equations, supplemented with $\left(s^{-}+s^{+}\right)(n+$ 1) $+2 n+s^{+}+2$ extra equations, resulting in the need for $s_{\eta}=s^{+}-s^{-}+1$ free parameters. This leads to a boundary value problem, which is coupled to a number of algebraic constraints for the equilibria and their stability. The boundary value problem can be solved by a collocation method as in Sec. 2.1.

Good starting conditions for Newton's method can be obtained as follows. For a homoclinic orbit, one can start from a nearby periodic solution 
with a sufficiently large period. Heteroclinic orbits can be approximated by using time integration or by using an extension of the method of successive continuation [18]. Details of the method, including a numerical study of the convergence, are presented in [65].

\section{Quasiperiodic tori}

In dynamical systems quasiperiodic solutions reside on invariant tori. In this section we describe a method to compute two-dimensional tori as periodic functions on the unit square. In particular we adapt the method of Schilder et al. [67], which uses a finite difference method to discretize the defining equation. Here we use a spectral collocation method that is well suited to delay equations.

A quasiperiodic solution $x(t)$ of (1) has two rationally independent frequencies $\omega_{1}, \omega_{2}$. Hence, there exists a function $y: \mathbb{R}^{2} \rightarrow \mathbb{R}^{n}$, which is $2 \pi$ periodic in both variables, such that $x$ can be written as $x(t)=y\left(\omega_{1} t_{1}, \omega_{2} t_{2}\right)$. Putting $y$ into (1) yields a first order delayed partial differential equation

$$
\begin{array}{r}
\frac{\partial}{\partial_{t_{1}}} y\left(t_{1}, t_{2}\right)+\frac{\omega_{2}}{\omega_{1}} \frac{\partial}{\partial_{t_{2}}} y\left(t_{1}, t_{2}\right)=\frac{1}{\omega_{1}} f\left(y\left(t_{1}, t_{2}\right), y\left(t_{1}-\omega_{1} \tau_{1}, t_{2}-\omega_{2} \tau_{1}\right), \ldots\right. \\
\left.\ldots, y\left(t_{1}-\omega_{1} \tau_{m}, t_{2}-\omega_{2} \tau_{m}\right), \eta\right),
\end{array}
$$

where $\omega_{1}, \omega_{2}$ are unknown frequencies. Because there are translational symmetries in both variables of $y$, two phase conditions have to be imposed on $y$ in order to fix a unique solution and determine the unknown frequencies. Assuming that we have a reference solution $u^{(0)}$ of $(32)$ at $\eta_{0}$, we formulate a condition that minimizes the distance of $u$ at $\eta$ from $u^{(0)}$, i.e.,

$$
\kappa\left(\theta_{1}, \theta_{2}\right)=\frac{1}{(2 \pi)^{2}} \int_{0}^{2 \pi} \int_{0}^{2 \pi}\left\|u\left(t_{1}+\theta_{1}, t_{2}+\theta_{2}\right)-u^{(0)}\left(t_{1}, t_{2}\right)\right\|_{2}^{2} \mathrm{~d} t_{1} \mathrm{~d} t_{2} .
$$

Taking the first derivative of $\kappa$ with respect to $\theta_{1}$ and $\theta_{2}$, the phase conditions become

$$
\begin{aligned}
& \frac{1}{(2 \pi)^{2}} \int_{0}^{2 \pi} \int_{0}^{2 \pi} \frac{\partial}{\partial t_{1}} u^{(0)}\left(t_{1}, t_{2}\right) u\left(t_{1}, t_{2}\right) \mathrm{d} t_{1} \mathrm{~d} t_{2}=0 \\
& \frac{1}{(2 \pi)^{2}} \int_{0}^{2 \pi} \int_{0}^{2 \pi} \frac{\partial}{\partial t_{2}} u^{(0)}\left(t_{1}, t_{2}\right) u\left(t_{1}, t_{2}\right) \mathrm{d} t_{1} \mathrm{~d} t_{2}=0 .
\end{aligned}
$$

In the case of time periodic systems only the second phase condition is necessary, since the phase in $t_{1}$ is fixed by the phase of the forcing. In addition to the phase conditions, we also need boundary conditions that guarantee the periodicity of $u$, that is,

$$
\begin{gathered}
u\left(0, t_{2}\right)=y\left(2 \pi, t_{2}\right) \quad \text { and } \\
u\left(t_{1}, 0\right)=y\left(t_{1}, 2 \pi\right), \quad \forall \quad t_{1}, t_{2} \in[0,2 \pi] .
\end{gathered}
$$




\subsection{Spectral collocation}

To obtain an approximation of the quasiperiodic solution the defining sets of equations can be solved with an appropriate numerical scheme and Newton's method. There are several different spectral collocation methods for partial differential equations that could be used to solve (32); for an introduction see Trefethen [76]. Here we use a method that was developed for computationally challenging hyperbolic equations such as the Navier-Stokes equation. The method is a multi-domain spectral collocation method called the staggered grid Chebyshev method, developed by Kopriva and Kolias [48].

The method is similar to the collocation of periodic solutions. It uses piecewise polynomials that are represented by their values at discrete points of a mesh, which is different from the mesh on which the equation is solved. We use a very simple domain subdivision of the area $[0,2 \pi] \times[0,2 \pi]$ that splits it into the rectangles

$$
D_{i, j}=\left[t_{1}^{i}, t_{1}^{i+1}\right] \times\left[t_{2}^{j}, t_{2}^{j+1}\right]
$$

where $\left\{0=t_{l}^{0}<t_{l}^{1}<\cdots<t_{l}^{L_{l}}=2 \pi\right\}$ with $l \in\{1,2\}$. On each rectangle $D_{i, j}$ we use the Lobatto points $\left(t_{1}^{i, p}, t_{2}^{j, q}\right)=\left(t_{1}^{i}+b_{1}^{p}\left(t_{1}^{i+1}-t_{1}^{i+1}\right), t_{2}^{i}+b_{2}^{q}\left(t_{2}^{j+1}-t_{2}^{j+1}\right)\right)$ to represent the solution

$$
u\left(t_{1}, t_{2}\right)=\sum_{p=0}^{d_{1}} \sum_{q=0}^{d_{2}} u\left(t_{1}^{i, p}, t_{1}^{j, q}\right) P^{i, j, p, q}\left(t_{1}, t_{2}\right),
$$

where $P^{i, j, p, q}$ are the Lagrange polynomials through the points $\left(t_{1}^{i, p}, t_{2}^{j, q}\right)$. The function $u$ is now completely determined by the values

$$
u_{i, j, p, q}:=u\left(t_{1}^{i, p}, t_{1}^{j, q}\right)
$$

which we consider identical if they represent the same point in $[0,2 \pi] \times[0,2 \pi]$. We also need to impose the boundary conditions, which are

$$
\begin{aligned}
& u_{0, j, 0, q}=u_{L_{1}-1, j, d_{1}, q}, \quad 0 \leqslant q<d_{2}, 0 \leqslant j<L_{2}-1 \\
& u_{i, 0, p, 0}=u_{i, L_{2}-1, p, d_{2}}, \quad 0 \leqslant p<d_{1}, 0 \leqslant i<L_{1}-1 \text { and } \\
& u_{0,0,0,0}=u_{L_{1}-1, L_{2}-1, d_{1}, d_{2}} .
\end{aligned}
$$

It is also possible to think of the piecewise polynomials as discontinuous in the interfaces and define mortar equations as in spectral penalty methods (see, e.g., Hesthaven [43]) instead of (34).

Equation (32) is solved on the grid

$$
\begin{aligned}
& \left(\hat{t}_{1}^{i, p}, \hat{t}_{2}^{j, q}\right)=\left(t_{1}^{i}+c_{1}^{q}\left(t_{1}^{j+1}-t_{1}^{j+1}\right), t_{2}^{i}+c_{2}^{q}\left(t_{2}^{j+1}-t_{2}^{j+1}\right)\right), \\
& 0 \leqslant i<L_{1}, 0 \leqslant j<L_{2}, 0 \leqslant p<d_{1}, 0 \leqslant q<d_{2},
\end{aligned}
$$




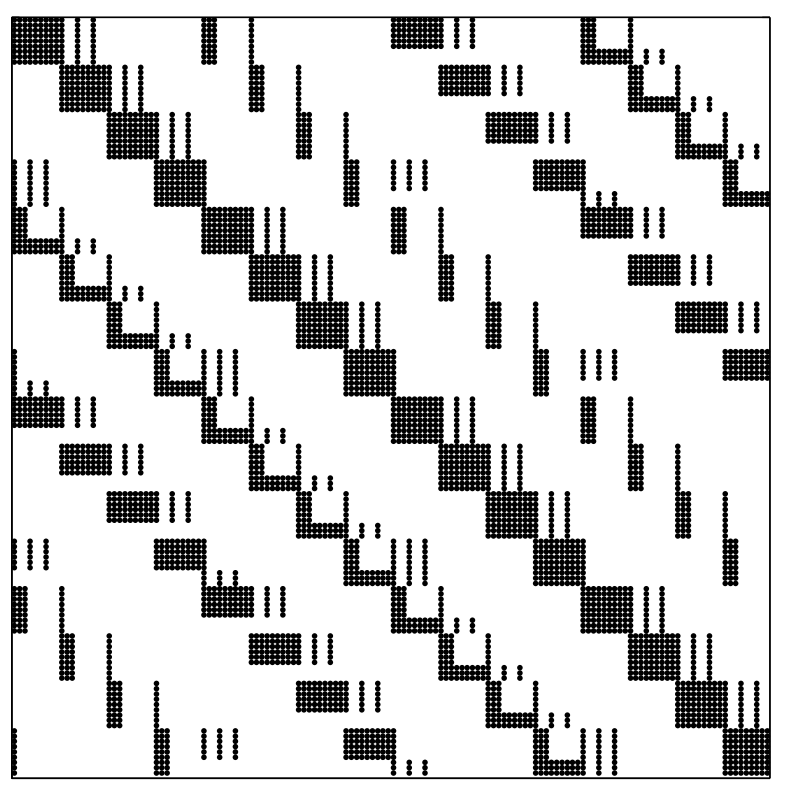

Fig. 3. Sparsity structure of the Jacobian of the discretized (32). The seemingly irregular pattern is due to the patching of the rectangles defined by (34); parameters are $L_{1}=L_{2}=4, d_{1}=d_{2}=3$ and $n=1$.

where $c_{1}^{p}, c_{2}^{q}$ are the Gauss points. Using the polynomial representation (33) of $u$ in (32) and evaluating at $\left(\hat{t}_{1}^{i, p}, \hat{t}_{2}^{j, q}\right)$ yields a large algebraic system that can be solved by Newton's method. The typical sparsity structure of the Jacobian of this discretized system is shown in Fig. 3, but without the borders accounting for the phase conditions.

\section{Further classes of delay equations}

We now briefly review continuation methods for systems that have nonconstant or derivative delay terms.

\subsection{State dependent equations}

We briefly describe how a DDE with state-dependent delay (sd-DDE) can be handled; we assume for simplicity that only one delay is present. An sd-DDE can be of the form

$$
\left\{\begin{array}{l}
\frac{\mathrm{d}}{\mathrm{d} t} x(t)=f_{1}(x(t), x(t-\tau(x(t)), \eta) \\
\tau(x(t))=g_{1}(x(t))
\end{array}\right.
$$


where $g_{1}: \mathbb{R}^{n} \rightarrow \mathbb{R}$ is a given (explicit) function of the solution $x(t)$, or it can be of the form

$$
\left\{\begin{array}{l}
\frac{\mathrm{d}}{\mathrm{d} x} x(t)=f_{2}(x(t), x(t-\tau(t)), \tau(t)) \\
\frac{\mathrm{d}}{\mathrm{d} t} \tau(t)=g_{2}(x(t), x(t-\tau(t)), \tau(t)),
\end{array}\right.
$$

where $g_{2}: \mathbb{R}^{n} \times \mathbb{R}^{n} \times \mathbb{R} \rightarrow \mathbb{R}$, and the delay is determined by a differential equation. We assume that all functions in (35) and (36) are sufficiently smooth and that theq delay is bounded, i.e., $0 \leq \tau(t) \leq r, \forall t$. Note that, using $x_{1} \equiv x$ and $x_{2} \equiv \tau$, equation (36) can be considered as a particular case of (35) with the extended state $x \equiv\left(x_{1}, x_{2}\right)$.

A steady-state solution of a sd-DDE is determined by the state $x$ and the delay $\tau$, i.e., the delay should be considered as a part of the solution. A steady-state solution $\left(x^{*}, \tau^{*}\right)$ of $(35)$ or $(36)$ can be computed by solving a (nonlinear) algebraic system. The local stability of steady-state solutions of sd-DDEs was studied in [14, 39]. It was shown, under natural assumptions on the right-hand side of the equation and on the delay function $\tau$, that generically the behaviour of the state-dependent delay $\tau$, except for its value $\tau^{*}$, has no effect on the stability, and that in the local linearization $\tau$ can be treated as a constant. Hence, to study the local stability of a steady state of (35) or (36), we linearize these equations at $x^{*}$ by setting $\tau \equiv \tau^{*}$. The resulting linearized equation is a DDE with constant delay, and the numerical procedures discussed in Sec. 1 can be used without changes.

The existence of periodic solutions for particular cases of sd-DDEs has been studied by several authors, in particular the existence of 'slowly oscillating periodic solutions'. The theory suggests that a Hopf bifurcation theorem holds; see, e.g., [57]. The stability of periodic solutions has only recently been studied; see, e.g., [40] for non-autonomous sd-DDEs. It was proven that the Fréchet derivative of the solution operator of the nonlinear sd-DDE with respect to initial data equals the solution operator of the linearized equation. Based on these results we linearize (35) and (36) around a (nonconstant) solution $\left(x^{*}(t), \tau^{*}(t)\right)$ as follows. Let $D_{j} f_{i}$ denote the derivative of $f_{1}$ with respect to its $j$-th argument, then

$$
\begin{aligned}
\frac{\mathrm{d}}{\mathrm{d} t} y(t) & =D_{1} f_{1}(s) y(t)-D_{2} f_{1}(s) \frac{\mathrm{d}}{\mathrm{d} t} x^{*}\left(t-\tau\left(x^{*}(t)\right)\right) \frac{\partial}{\partial x} \tau\left(x^{*}(t)\right) y(t) \\
& \left.+D_{2} f_{1}(s) y\left(t-\tau\left(x^{*}(t)\right)\right)\right)
\end{aligned}
$$

with $s=\left(x^{*}(t), x^{*}\left(t-\tau\left(x^{*}(t)\right)\right)\right)$, respectively, and

$$
\left\{\begin{aligned}
\frac{\mathrm{d}}{\mathrm{d} t} y_{1}(t) & =D_{1} f_{2}(s) y_{1}(t)+D_{2} f_{2}(s) y_{1}\left(t-\tau^{*}(t)\right)-D_{2} f_{2}(s) \frac{\mathrm{d}}{\mathrm{d} t} x^{*}\left(t-\tau^{*}(t)\right) y_{2}(t) \\
& +D_{3} f_{2}(s) y_{2}(t) \\
\frac{\mathrm{d}}{\mathrm{d} t} y_{2}(t) & =D_{1} g_{2}(s) y_{1}(t)+D_{2} g_{2}(s) y_{1}\left(t-\tau^{*}(t)\right)-D_{2} g_{2}(s) \frac{\mathrm{d}}{\mathrm{d} t} x^{*}\left(t-\tau^{*}(t)\right) y_{2}(t) \\
& +D_{3} g_{2}(s) y_{2}(t)
\end{aligned}\right.
$$

with $s=\left(x^{*}(t), x^{*}\left(t-\tau^{*}(t)\right), \tau^{*}(t)\right)$. These linearized equations contain a timedependent (no longer state-dependent) delay. If the coefficients in the linear equation are smooth and periodic (with period $T$ ) and the delay function is 
smooth, then the solution operator over the period $T$ (over an interval $m T$ if $\tau_{m}>T$ and $\left.m T \geq \tau_{m}, \tau_{m}=\max _{t \in[0, T]} \tau(t)\right)$ is compact [36].

A periodic solution can be computed by solving a two-point boundary value problem in time, similar to (13), but in case of (36) the additional equation $\tau(0)=\tau(T)$ must be imposed. The solution of these boundary value problems by collocation and the computation of the Floquet multipliers is conceptually equal to the procedure outlined in Sec. 2.

\subsection{Collocation schemes for equations of neutral type}

We summarise basic results on two collocation schemes that were proposed in Barton et. al. [5]. Here we consider the simple equation of neutral type

$$
\dot{x}(t)=f(x(t), x(t-\tau), \dot{x}(t-\tau), \eta) .
$$

The collocation scheme of Sec. 2.1 discretizes (38) by substituting the collocation polynomials and evaluating at the collocation points. In the Jacobian matrix of this discretized system the second derivatives of the polynomials appear. This reduces the accuracy by an order, which is only $O\left(h^{m}\right)$. This drop in the order of convergence is apparent in the examples of [5]. To remedy the situation (38) can be transformed into an ODE coupled to a difference equation

$$
\begin{aligned}
& \dot{x}(t)=y(t) \\
& y(t)=f(x(t), x(t-\tau), y(t-\tau) ;
\end{aligned}
$$

see [5]. Applying the collocation scheme of Sec. 2.1 to this system does not introduce second order derivatives in the Jacobian matrix and, hence, a better convergence can be expected. The numerical experiments in [5] show a convergence rate of $O\left(h^{m+1}\right)$. In [5] the Gauss-Legendre points were used in the collocation scheme, together with a periodic boundary condition on the algebraic part, but other approaches are possible for delay differential algebraic equations.

\section{Software packages}

Several software packages exist for simulation (time integration) of delay differential equations, including ARCHI [62], DKLAG6 [15], RADARS [?] and XPPAUT [28]. Probably the earliest computer program specifically designed for DDEs has been published by Hassard [41], namely BIFDD which allows a normal form analysis of Hopf bifurcation points. XPPAUT by Ermentrout [28] allows a limited stability analysis of steady-state solutions of DDEs using the approach described in [54].

By contrast, the software packages DDE-BIFTOOL and PDDE-ConTimplement numerical continuation DDEs as introduced in the previous sections. In this section we describe the functionality of these numerical tools. 


\subsection{DDE-BIFTOOL}

The package DDE-BIFTOOL consists of a collection of MATLAB-routines for the numerical continuation and bifurcation analysis of systems of DDEs with multiple discrete delays, which may be fixed or state-dependent; for detailed instructions we refer to the user manual [26]. This software allows one to compute branches of steady-state solutions and steady-state fold and Hopf bifurcations with continuation. Given an equilibrium, it allows to approximate the right-most, stability-determining roots of the characteristic equation, which can be further corrected with Newton's method. Periodic solutions and their Floquet multipliers can also be computed by collocation with adaptive mesh selection. Branches of periodic solutions can be continued starting from a previously computed Hopf point or from an initial guess of a periodic solution profile. For DDEs with constant delays, also connecting orbits (both homoclinic and heteroclinic solutions) can be computed. The numerical methods that are used in the software are as detailed in the previous sections.

DDE-BIFTOOL has no graphical user interface, but a number of routines are provided to plot solution, branch and stability information. Furthermore, automatic detection of bifurcations is not supported. Instead, the evolution of the characteristic roots or the Floquet multipliers can be computed along solution branches, which allows the user to detect and identify bifurcations using an appropriate visualisation. Starting points for branch switching at bifurcations on branches of steady-state and periodic solutions can be generated, as well as starting solutions for homoclinic solutions close to periodic solutions.

Several extensions or 'add-ons' have been developed. We mention here a Mathematica program written by Pieroux that allows the automatic generation of the system definition files with symbolically obtained derivatives, software written by Green for the computation of 1D unstable manifolds in DDEs [33], and the extension by Barton for equations of neutral type [5].

\subsection{PDDE-CONT}

PDDE-ConT implements the numerical methods described in Sec. 2. It is written in $\mathrm{C}++$ with the use of linear algebraic packages UMFPACK [16],LAPACK [?] and ARPACK [?]. The software has a command line interface and a graphical user interface together with a basic plotting facility.

PDDE-Cont can continue periodic solutions of delay equations that are in the form

$$
y^{\prime}(t)=f\left(t, y(t), y\left(t-\tau_{1}(t)\right), y\left(t-\tau_{2}(t)\right), \ldots, y\left(t-\tau_{m}(t)\right), \eta\right) .
$$

The right-hand side $f$ and the delays $\tau_{j}$ can be either $T$-periodic or time independent. The software does not have any algorithms to continue equilibria apart from the obvious fact that an equilibrium can be considered as 
a constant periodic solution. Bifurcations of periodic solutions can be continued in two parameters by using test functions as described in Sec. 3, but PDDE-CONT cannot switch branches automatically. For detailed instructions see the user manual [73]. Note that PDDE-ConT can be used together with DDE-BIFTOOL by converting the results between the two packages.

Due to the low level programming approach, the performance of PDDECONT is significantly better than that of DDE-BIFTOOL (which runs under MATLAB). Furthermore, PDDE-CONT uses sparse-matrix alogorithms that require less memory, so that problems of relatively high dimension can be tackled. The resulting large bordered linear systems (see Sec. 2.1) are solved by using bordering techniques from [30, 31]. The large sparse matrix without borders is factorized by UMFPACK and the whole system is solved using the BEMW method [31].

\section{Examples of numerical bifurcation analysis of DDEs}

In this section we illustrate the performance of the numerical techniques described in the previous sections with examples of DDE models of a number of physical and biological phenomena.

\subsection{DDE-PDE model of a laser with optical feedback}

A longitudinally single-mode semiconductor laser subject to conventional optical feedback and lateral carrier diffusion can be modeled by the hybrid DDEPDE system

$$
\begin{aligned}
\frac{\mathrm{d} A(t)}{\mathrm{d} t}= & (1-\mathrm{i} \alpha) A(t) \zeta(t)+\eta A(t-\tau) e^{-\mathrm{i} \phi}-\mathrm{i} b A(t), \\
T \frac{\partial Z(x, t)}{\partial t}= & d \frac{\partial^{2} Z(x, t)}{\partial x^{2}}-Z(x, t)+P(x) \\
& -F(x)(1+2 Z(x, t))|A(t)|^{2} .
\end{aligned}
$$

Here the complex scalar variable $A(t)$, represents the amplitude of the electrical field $E(t)=A(t) e^{i b t}$, and real $Z(x, t), x \in[-0.5,0.5]$, represents the carrier density [77], The functions $\zeta(t), P(x)$ and $F(x)$ are specified in [77]. Continuous-wave solutions, called 'external cavity modes' (ECMs) can be computed as steady-state solutions of (41)-(42), augmented with a scalar condition for the unknown $b$ and an extra scalar constraint to remove the $S^{1}$-symmetry. Zero Neumann boundary conditions for $Z(x, t)$ are imposed at $x= \pm 0.5$. In the computations the time variable is rescaled so that one time unit represents 1/1000 of a second. NOTE: this must be checked !!! The symmetry about $x=0$ is exploited by considering only the interval $[0,0.5]$. Splitting (41) into real and imaginary part and discretizing (42) in space with a second order central difference formula with constant stepsize $\Delta x=0.5 / 128$. 


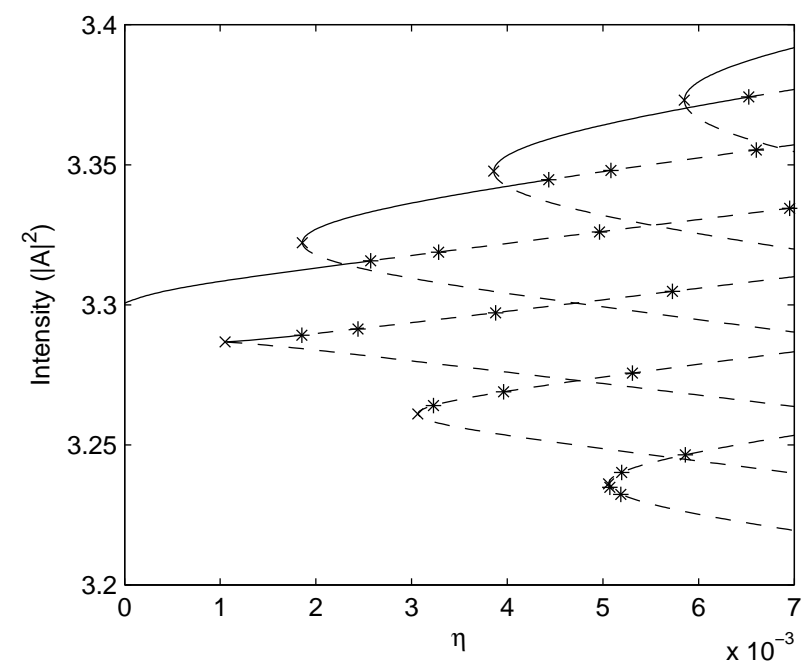

Fig. 4. Bifurcation diagram in the plane of intensity $|A|^{2}$ vs. feedback strength $\eta$ of steady-state solutions of (41)-(42) for $\alpha=3, \phi=0, T=1000, d=1.68 \times 10^{-2}$ and $\tau=1000$. Stable solutions are drawn as solid curves and unstable solutions as dashed curves; also shown are saddle-node bifurcations $(\times)$ and Hopf bifurcations $(*)$. Reproduced from K. Verheyden, K. Green, and D. Roose, Numerical stability analysis of a large-scale delay system modeling a lateral semiconductor laser subject to optical feedback, Phys. Rev. Lett. 69(3) (2004) 036702 (C) 2004, with permission from the American Physical Society.

Figure 4 shows the bifurcation diagram of steady-state solutions of (41)(42) with $\alpha=3, \phi=0, T=1000, d=1.68 \times 10^{-2}$ and $\tau=1000$, obtained by continuation with DDE-BIFTOOL, with the feedback strength $\eta$ as the parameter. The diagram shows several branches of steady-state solutions arising from saddle-node bifurcations. During continuation the right-most characteristic roots are computed and monitored, allowing for the detection of Hopf bifurcation points along these branches.

Figure 5 shows the characteristic roots at the moment of the first Hopf bifurcation of middle branch at $\eta \approx 2.5717 \times 10^{-3}$. Since the imaginary part of the right-most pair of characteristic roots is large, the system presents a challenging test case for characteristic root calculation with DDE-BIFTOOL.

Approximations to the characteristic roots were obtained by computing the eigenvalues of the matrix approximation to the solution operator as described in [80] with a 6th order LMS method, optimized to retain the stability properties of the linearized equation. The steplength $h$ in the LMS method is automatically determined to ensure that all characteristic roots with real part larger than $r=-1$ (threshold specified by the user) are approximated accurately. This leads to the discretization of the delay interval with an equidistant mesh of 27 points. The resulting eigenvalue problem has 


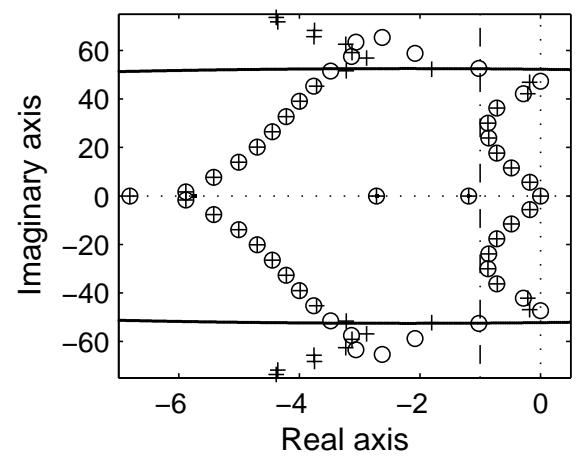

Fig. 5. Characteristic roots at the Hopf point of $(41)-(42) \eta \approx 2.5717 \times 10^{-3}$ from Fig. 4. Shown are approximations of characteristic roots with real part larger than $r=-1$ derived from the eigenvalues of the discretization of the solution operator by the 6th order special-purpose LMS method $(+)$, and their corrections by Newton's method (o). Reproduced from K. Verheyden, T. Luzyanina, and D. Roose, Efficient and reliable stability analysis of solutions of delay differential equations, Proceedings of 2006 International Conference on Nonlinear Science and Complexity, 109-120 (c) 2007, with permission from World Scientific Publishing.

Table 1. The computational cost of four algorithms based on a pseudo-spectral discretization using polynomials of degree $p=32$ to find the right-most characteristic roots shown in Fig. 5.

\begin{tabular}{|l||cc|cc|}
\hline \multicolumn{1}{|c||}{} & \multicolumn{2}{c|}{$\mathcal{A}$} & \multicolumn{2}{c|}{$\mathcal{S}(h)$} \\
\cline { 2 - 5 } & Right-most & Shift-Invert & Forward & Backward \\
\hline \hline CPU time (seconds) & 106.2 & 94.1 & 103.4 & 55.5 \\
\# matrix-vector products & 6528 & 4951 & 6254 & 3146 \\
\hline
\end{tabular}

dimension $131 \times 27=3537$, which is large but can still be solved by using the QR-method. These approximations are subsequently corrected by Newton's method applied to (8). The approximate characteristic roots shown in Fig. 5 were derived from the eigenvalues of the discretization of the solution operator, and their corrections by Newton's method.

For this example a comparison of the computation of the characteristic roots using the pseudo-spectral discretizations of the infinitesimal generator $\mathcal{A}$ and of the solution operator is presented in [81]. In both cases, a polynomial of degree $p=32$ is used, so that the linear eigenvalue problems have size $n(p+1)=131 \times 33=4323$. Table 1 shows the computational cost of the four methods.

To solve the linear eigenvalue problem resulting from the pseudo-spectral discretization of the infinitesimal generator, the MATLAB function eigs function is used to compute the right-most 30 eigenvalues with a requested tol- 
erance of $10^{-8}$ (results indicated with 'Right-most'). Note that eigs uses Arnoldi's method with implicit restart, and this method does not require the explicit construction of the matrix. For the results indicated with 'ShiftInvert', eigs is used in conjunction with the shift-invert technique and returns the eigenvalues $\lambda$ closest to the shift $\left\|A_{0}\right\|+\left\|A_{1}\right\| \approx 4528.5$, as proposed in [9]. The pseudo-spectral discretization of the solution operator $\mathcal{S}(h)$ leads to two algorithms, called forward and backward variants in [81]. The steplength $h$ is chosen to be $10^{-4}$ for the forward and $10^{-3}$ for the backward variant, respectively.

The accuracy of the computed characteristic roots is similar for the four methods. For example, the roots $-0.285 \pm i 11.8$ are computed by the four algorithms with a relative error between $6.510^{-12}$ and $2.410^{-14}$. The accuracy is lower for the eigenvalues with large imaginary part (the relative error on the pure imaginary eigenvalues $\pm i 47.7$ is $\approx 10^{-4}$ for the backward variant, and $\approx 710^{-6}$ for the three other algorithms. The exponential convergence with respect to the degree $p$ has been confirmed by numerical experiments.

\subsection{The Mackey-Glass equation}

The equation

$$
\dot{x}(t)=a x(t)+b \frac{x(t-\tau)}{1+x^{10}(t-\tau)} .
$$

models the regeneration of white blood cells [55], and it is today widely known as the Mackey-Glass equation. Although it is a simple equation, not much is known about its solution structure.

The three equilibria of (43), i.e., $x_{1}=0$ and $x_{2,3}=\sqrt[10]{-(a+b) / a}$ are connected to each other at $a=-b$ by a supercritical pitchfork bifurcation. The nonzero solutions can lose their stability in a Hopf bifurcation along the curves in parameter space given by

$$
\begin{aligned}
& a=-\arccos \left(-d^{-1}\right) \frac{1}{\tau \sqrt{d^{2}-1}}, \\
& b=\frac{10 a}{d-9},
\end{aligned}
$$

where $|d| \geq 1$. Hopf bifurcations for $d>1$ are supercritical, so they give rise to stable periodic solutions. These periodic solutions bifurcate further of several period doublings, which then leads to chaotic motion. It was demonstrated in [37] that chaos arises due to the transverse intersection of the two-dimensional unstable and infinite dimensional stable manifold of this periodic solution. We remark that some square shaped solutions of large period can be obtained by singularly perturing a map to give

$$
\varepsilon \dot{x}(t)=a x(t)+b \frac{x(t-1)}{1+x^{c}(t-1)},
$$

where $\varepsilon \rightarrow 0$ and $\varepsilon \tau=1$; see [56] for details. 


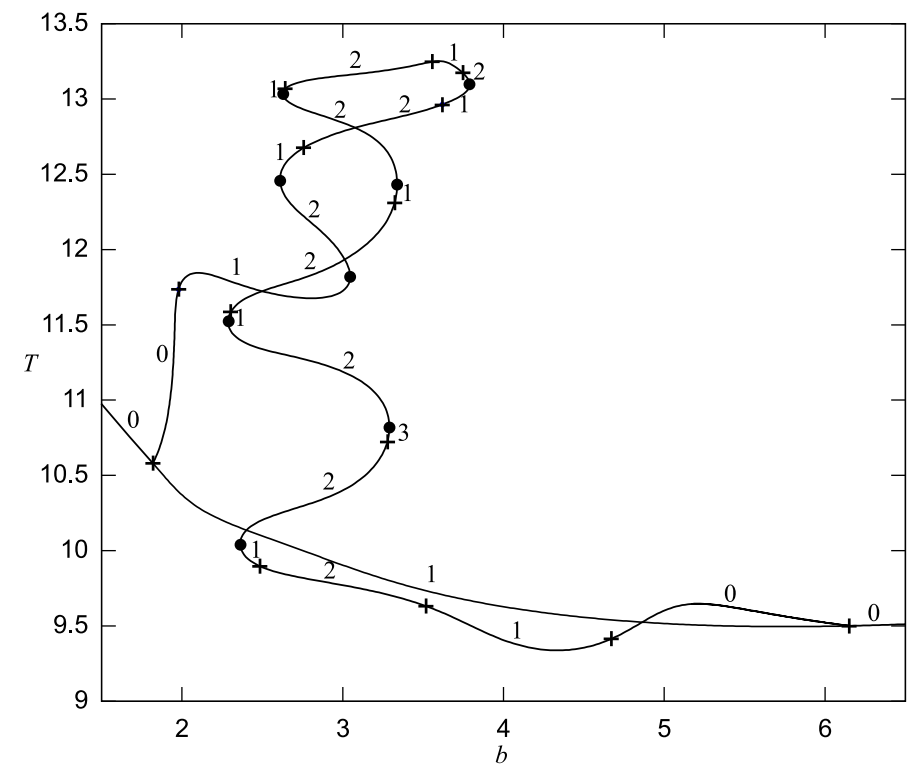

Fig. 6. Bifurcation diagram showing period-two solutions of (43) for fixed $a=$ -1.2158 . Fold bifurcations are denoted by dots while period doublings are denoted by + ; the numbers of unstable characteristic multipliers are indicated along the different branches.

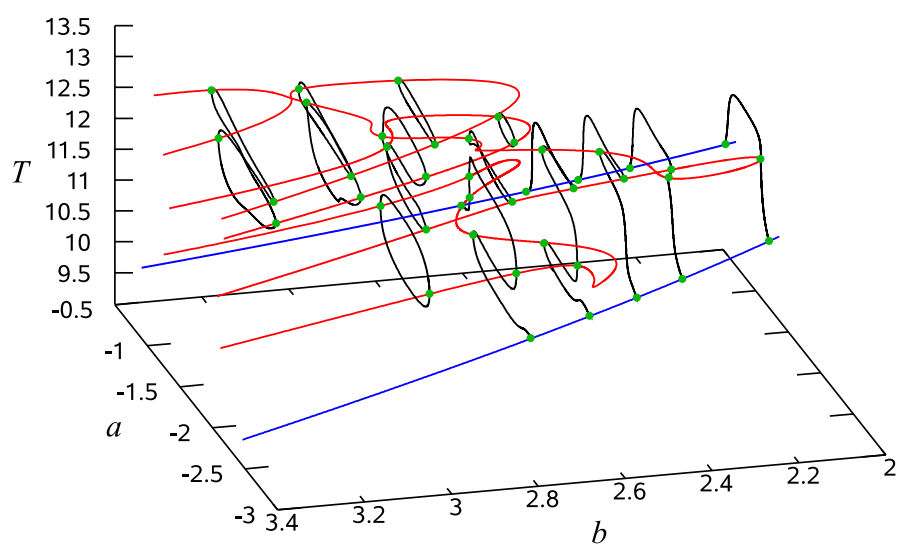

Fig. 7. The structure of the period-two solutions of (43) over the $(a, b)$-plane; shown are solution branches (black curves), their fold bifurcations (green dots), perioddoubling bifurcations of the period-one solution (blue curves) and fold bifurcations of period-two solutions (red curves). 
Here we analyze the period-two solutions bifurcating from the period-one solutions that in turn can be related to the supercritical Hopf bifurcation of the equilibrium. These solutions form a complicated branch structure that is challenging to compute. In Fig. 6 the bifurcation diagram for $a=-1.2158$ and $\tau=2$ is shown. As $b$ varies the solution undergoes several fold and period-doubling bifurcations. These solutions are almost all unstable, and so they cannot be found by simulation; the number of unstable characteristic multipliers is shown along the branches in Fig. 6. Furthermore, the periodone solution branch is included, but with twice period so that it matches up with the branch of period-two solutions.

By investigating the fold bifurcations and computing several branches of solutions we can obtain a fairly complete picture of the structure of periodic solutions. Figure 7 shows this structure for the fixed at delay $\tau=2$, where we plot the period $T$ of the solutions as a function of the parameters $a$ and $b$.

\subsection{Traffic model with driver reaction time}

The traffic model in Orosz et al. $[61,60]$ describes the dynamics of $N$ cars on a circular track. Each car has a velocity $v_{i}$ and an associated headway $h_{i}$ defined as the distance to the car in front. The headways $h_{i}$ are calculated from the velocities as

$$
\dot{h}_{i}(t)=v_{i+1}(t)-v_{i}(t)
$$

Because of the circular track, we assume that $v_{N+1}=v_{1}$ and $h_{N}=L-$ $\sum_{i=1}^{N-1} h_{i}$. Each car tries to reach its optimal velocity, which is a function of the headway that can be expressed as

$$
\dot{v}_{i}(t)=\beta\left(V\left(h_{i}(t-1)-v_{i}(t)\right),\right.
$$

where $\beta$ is the sensitivity to velocity differences. Due to the reaction time of driver, a delayed value of the headway is used in the model. The optimal velocity is a function of the headway, and it is modelled by the optimal velocity function

$$
V(h)=\left\{\begin{array}{cc}
0 & 0 \leq h \leq 1, \\
v^{0} \frac{(h-1)^{3}}{1+(h-1)^{3}} \quad h>1 .
\end{array}\right.
$$

By making use of the algebraic condition for $h_{N}$ one can reduce the dimension of system (44)-(45) by one to $2 N-1$.

In this section we consider $N=17$ cars, which is the largest number of cars that was considered and (partially) analyzed in [60]. Our starting point is the steady-state solution of the model, which corresponds to equal headways and equal car velocities and so is given by

$$
h_{i}^{*}=L / N, \quad v_{i}^{*}=V\left(h_{i}^{*}\right) .
$$




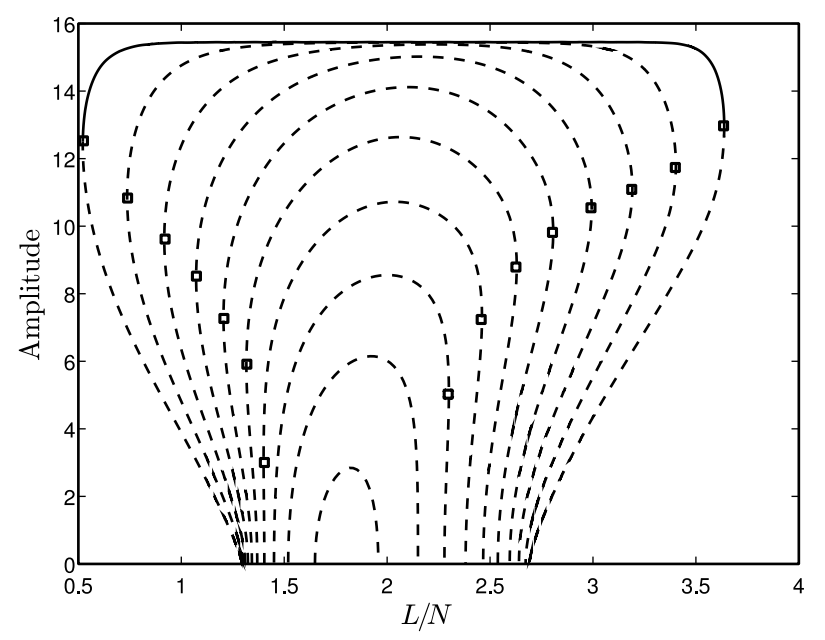

Fig. 8. Periodic solution branches of (44), (45). Unstable solutions are denoted by dashed lines, continous lines refer to stable solutions and boxes denote fold bifurcations.

The steady-state undergos several Hopf bifurcations from which branches of periodic solutions arise; they are shown in Fig. 8 as computed with PDDECONT for typical parameter values of $\beta=1$ and $\tau=2$ as a function of the average headway $L / N$. Note how all branches of periodic solutions feature folds and connect pairs of subcritical Hopf bifurcations. The outer-most branch is stable between the folds, which shows that there is bistability between stable periodic solutions (a traffic jam) and the stable steady-state (uniform flow of cars). The other branches of periodic solutions remain unstable throughout, but the outer-most of them has all its unstable Floquet multipliers very close to one (for $l / N$ around 2), which means that the corresponding periodic solution can be observed as long transients. Physically, these transients are traffic jams; they move towards each other and eventually either merge with the stable traffic jam or disperse [60].

Figure 9 shows the curves of fold bifurcations for $N=17$ cars in the $(L / N, \beta)$-plane. The plot also shows some Hopf bifurcation curves (dashdotted lines) and points of degenerate Hopf bifurcations (+); the regions of bistability are highlighted in gray. In [60] a similar image was computed for $N=9$ cars with DDE-BIFTOOL by performing one-parameter continuations in $L / N$ for many values of fixed $\beta$ to find the fold bifurcations. (The locus of Hopf bifurcations is actually known analytically.) As this approach is very time consuming, we used PDDE-ConT instead, which is able to follow the fold bifurcation curves directly in two parameters. 


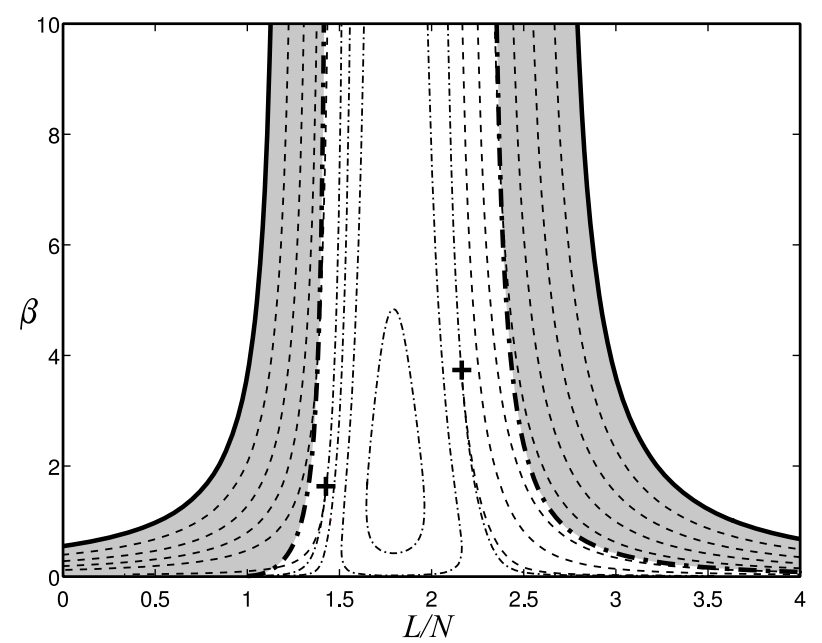

Fig. 9. Hopf and fold bifurcation curves of (44)-(45). The gray regions are bistable, where a stable equilibrium coexists with a stable periodic solution. Between the gray regions the equilibrium is unstable, while outside the gray regions the equilibrium is globally stable.

\subsection{Chatter motion in milling}

Cutting processes are often subject to the so-called regenerative effect [72], which comes from the fact that a cutting tool always cuts a surface that was produced by the same tool some time ago. The cutting forces nonlinearly depend on the chip geometry, which in turn depends on the current and a delayed tool position. The underlying dynamics of the tool can be considered to be linear and, hence, the nonlinearity comes from the geometry of the chip forming and the cutting force only. There is a vast literature on the dynamics of machining that mainly focuses on the stability of steady cutting; see, e.g., [45]. However, there are only few papers on the nonlinear dynamics and they employ either analytical methods [71] or simulation [3].

Machining processes are inherently non-smooth, because there is the possibility of a loss of cutting force when the tool leaves the work piece. This poses some challanges, although in some cases one can approximate the equations of motion with a smooth system. In the case of turning, which is an autonomous process, DDE-Biftool was used in [22]. Here we summarize the results in [74], where a milling problem was investigated with PDDE-CONT.

The equation of motion of the non-smooth milling problem reads

$$
\begin{aligned}
& \ddot{x}(t)+2 \xi \dot{x}(t)+x(t)=g(t) \hat{w}(\cos 2 \pi t / T+0.3 \sin 2 \pi t / T) \times \\
& \times\left[H(1+x(t-2 T)-x(t-T)) F_{c}((1+x(t-T)-x(t)) \sin 2 \pi t / T)\right. \\
& \left.+H(x(t-T)-x(t-2 T)-1) F_{c}((2+x(t-T)-x(t)) \sin 2 \pi t / T)\right],
\end{aligned}
$$



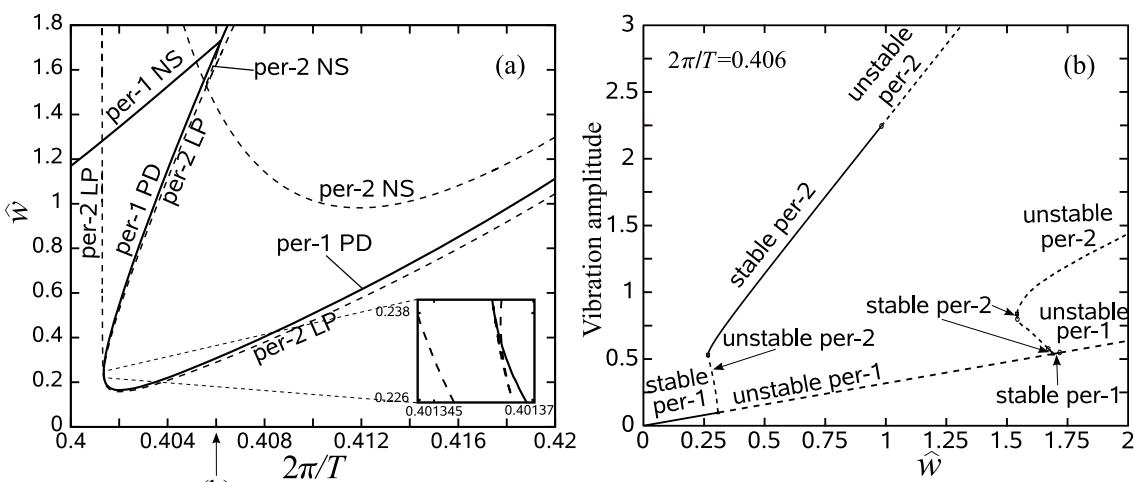

(b)

Fig. 10. Stability chart of (46) in the cutting speed and chip-width parameter plane (a); the relative damping is $\zeta=0.0038$ and the tool cuts continuously for a time of $10.82 \%$ of every period. Panel (b) shows a bifurcation diagram for fixed cutting speed; the fold bifurcations of the periodic solutions are due to the non-smooth dynamics of the system.

where $F_{c}$ is a nonlinear cutting force function, usually modelled with the power law $F_{c}(x)=4 \hat{w} / 3 x^{3 / 4}$, and $H$ is the Heaviside function. The function $g$ is a $T$-periodic windowing function that changes its value once in a period between 0 and 1 depending on whether the tool is cutting the material. The two important parameters are the period $T$, which is inversely proportional to the spindle speed and the dimensionless chip width $\hat{w}$.

In order to conduct a numerical bifurcation analysis of the system with PDDE-ConT, the Heaviside function $H(z)$ is replaced by the smoothed function $(1+\tanh (C z)) / 2$ with a sufficienly large value of $C$. Equation (46) has a unique $T$-periodic solution which represents steady cutting and can lose its stability either at a Neimark-Sacker or at a period-doubling bifurcation. Figure 10(a) shows the bifurcation diagram where these bifurcation curves are shown as solid curves. The period doublings may be subcritical or supercritical; see the bifurcation diagram for a fixed cutting speed in Fig. 10(b). Fold and Neimark-Sacker bifurcation curves of the period-two solutions have been continued in two parameters, and they are shown in Fig. 10(a) as dashed curves. These numerical results were compared to experimental data in [74].

Quasiperiodic solutions arising at a Neimark-Sacker bifurcation can be computed with the technique described in Sec. 5. A branch of invariant quasiperiodic tori was continued with PDDE-ConT until the model loses its physical validity. During the continuation the rotation number $\frac{\omega_{2}}{\omega_{1}}$ was kept constant and $\hat{w}$ and $T$ served as free parameters. The resulting curve of quasiperiodic solutions is shown in Fig. 11(a). Since $T$ varies only slightly during the continuation, the dependence on the period is not shown in the bifurcation diagram. One of the invariant tori along the branch (near where the model 

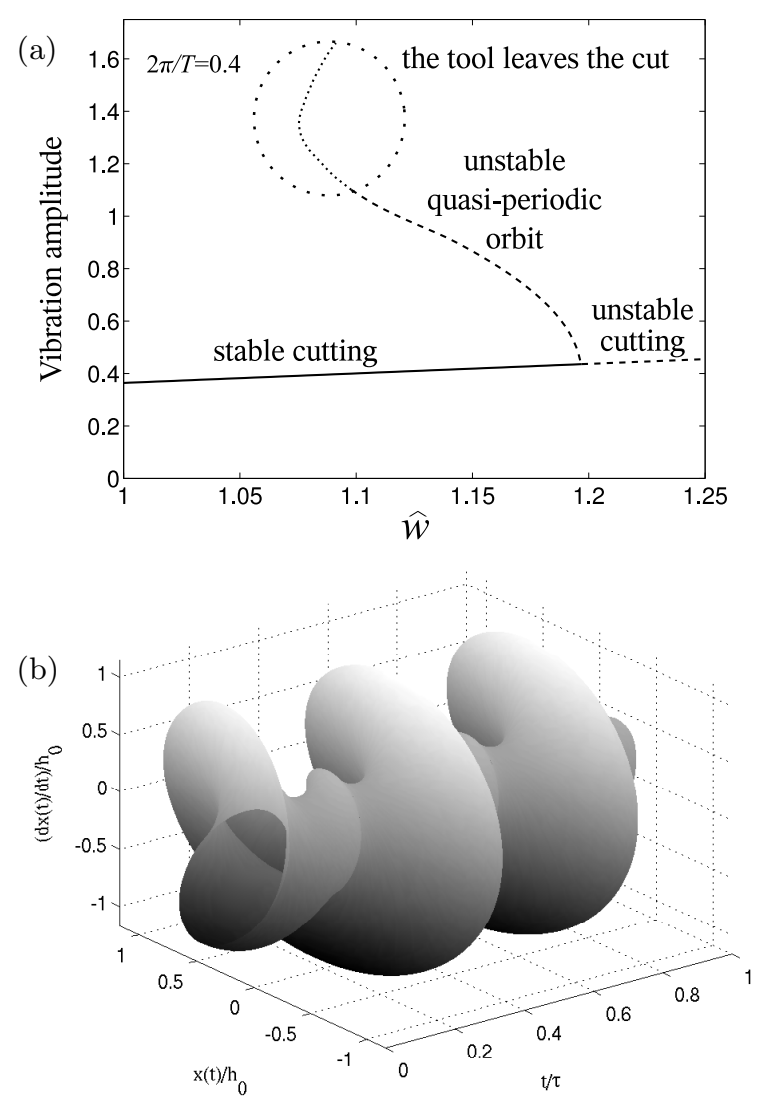

Fig. 11. A branch of quasiperiodic solutions for a cutting speed of $2 \pi / T=0.4$ (a) and an invariant torus (b) for a point on the branch just before the tool leaves the work piece and (46) becomes invalid.

loses its validity) is shown in Fig. 11(b). The computation of further quasiperiodic solutions reveals that this system has very narrow Arnol'd tongues in the region above the Neimark-Sacker curve in Fig. 10(a).

\subsection{A laser with filtered optical feedback}

One main objective for studying laser dynamics is to find regions of parameter values, where a constant amplitude coherent light is produced. In many laser systems delay is an important feature. It arises due to the finite travel time of light between components of the system and may lead to different types of dynamic behaviour including chaos; see, e.g., [47]. The numerical tools introduced in this chapter are very well suited for the study of nonlinear dynamics in lasers with delayed optical feedback; see also [49]. 
In this section we summarize some results of Erzgräber et. al. [29], who investigate a DDE model of a semiconductor laser with filtered optical feedback of the form

$$
\begin{aligned}
\frac{\mathrm{d} E}{\mathrm{~d} t} & =(1+i \alpha) N(t)+\kappa F(t), \\
T \frac{\mathrm{d} N}{\mathrm{~d} t} & =P-N(t)-(1+2 N(t))|E(t)|^{2}, \\
\frac{\mathrm{d} F}{\mathrm{~d} t} & =\Lambda E(t-\tau) \mathrm{e}^{-i C_{p}}+(i \Delta-\Lambda) F(t),
\end{aligned}
$$

where the variable $E$ is the complex optical field, $N$ is the (real-valued) population inversion of the laser, and $F$ is the complex optical field of the filter. The material properties of the laser are given by the linewidth enhancement factor $\alpha$, the electron lifetime $T$ and the pump rate $P$. The coupling of the laser with the filter is controlled by the parameter $\kappa$, while $\tau$ is the time that the light spends in the external feedback loop. The dynamics of the filter is modelled by (49). The feedback phase $C_{p}$ is the phase difference between the laser and the filter fields, and $\Delta$ is the detuning between the filter center frequency $\Omega_{F}$ and the solitary frequency $\Omega_{0}$ of the laser. Hence, $C_{p}=\Omega_{0} \tau$ and $\Delta=\Omega_{F}-\Omega_{0}$.

The laser equations (47)-(49) exhibit a rotational symmetry (rotation of both $E$ and $F$ over any angle) that is important for the types of solutions that are supported. It also needs to be dealt with in the continuation to ensure that solutions are isolated. The idea is to consider solutions of the form

$$
(E(t), N(t), F(t))=\left(A(t) \mathrm{e}^{i b t}, N(t), B(t) \mathrm{e}^{i b}\right) .
$$

By putting this ansatz into (47)-(49) we obtain the new system

$$
\begin{aligned}
\frac{\mathrm{d} A}{\mathrm{~d} t} & =(1+i \alpha) N(t) A(t)-i b A(t)+\kappa B(t) \\
T \frac{\mathrm{d} N}{\mathrm{~d} t} & =P-N(t)-(1+2 N(t))|A(t)|^{2}, \\
\frac{\mathrm{d} B}{\mathrm{~d} t} & =\Lambda A(t-\tau) \mathrm{e}^{-i\left(C_{p}+b \tau\right)}+(i \Delta-\Lambda-i b) B(t) .
\end{aligned}
$$

Note that the stability of this transformed system does not differ from the the stability of $(47)-(49)$, because the norm of $(E(t), N(t), F(t))$ is the same as the norm of $(A(t), N(t), B(t))$. System (50)-(52) still has the same rotational symmetry, but the equations are now in a form can be dealt with in continuation.

The primary interest is in the so-called external filtered modes (EFMs), which are single frequency periodic solutions of (50)-(52) that are characterized by fixed $A(t)=A_{s}, N(t)=N_{s}$ and $B(t)=B_{s}$. EFMs were extensively studied analytically [59] and with numerical continuation [29]. In order to determine an EFM uniquely one needs to fix the phase, which can be done, 

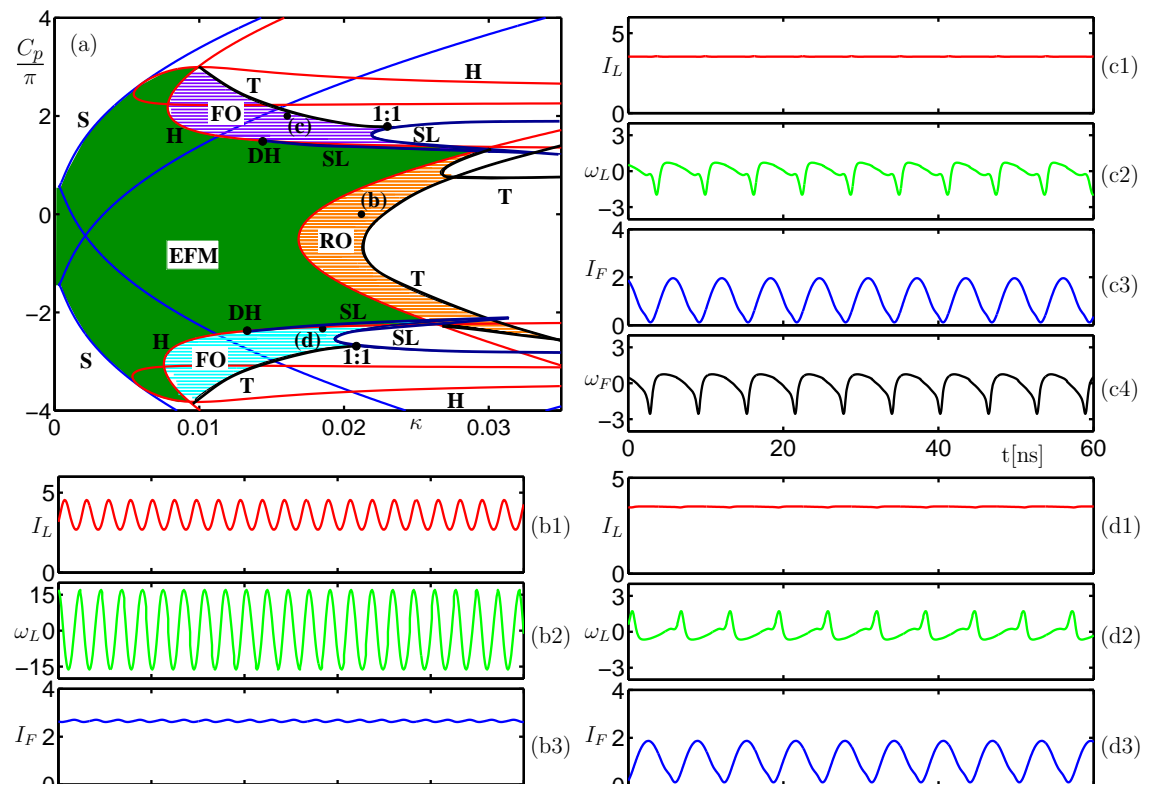

Fig. 12. Panel (a) shows the bifurcation diagram in the $\left(\kappa, C_{p}\right)$-plane of $(47)-(49)$ for $\Delta=0, \alpha=5.0, T=100, P=3.5, \tau=500$ and $\Lambda=0.007$. EFMs are stable in the green region, which is bounded by curves of saddle-node bifurcations (blue) and Hopf bifurcations (red). Panels (b1)-(b4) show an example of relaxation oscillations, while panels (c1)-(c4) and panels (d1)-(d4) are examples of frequency oscillations; plotted are the laser intensity $I_{L}$, its frequency $\omega_{L}$, the filter intensity $I_{F}$, and its frequency $\omega_{F}$. The stability regions of the different oscillations are shown in panel (a) in orange, purple and light blue, respectively. Reproduced from H. Erzgräber, B. Krauskopf and D. Lenstra, SIAM J. Appl. Dyn. Sys. 6(1) (2007) ??-?? (C) 2007, with permission from the Society for Industrial and Applied Mathematics.

for example, by setting $\operatorname{Re}\left(E_{s}\right)=0$ and treating $b$ as a variable. Figure 12(a) shows a bifurcation diagram in the $\left(\kappa, C_{p}\right)$-plane that was computed with DDE-BIFTOOL in this way. EFMs are stable in the green region; they are born in saddle-node bifurcations (blue curves) and lose their stability in Hopf bifurcations (red curves).

At Hopf bifurcations periodic solutions arise whose continuation requires a new phase condition [35]. Let us introduce the symmetry group

$$
G(\theta)=\left(\begin{array}{ccc}
\mathrm{e}^{i \theta} & 0 & 0 \\
0 & 1 & 0 \\
0 & 0 & \mathrm{e}^{i \theta}
\end{array}\right), \quad \mathfrak{G}=\left.\frac{\mathrm{d} G(\theta)}{\mathrm{d} \theta}\right|_{\theta=0}=\left(\begin{array}{lll}
i & 0 & 0 \\
0 & 0 & 0 \\
0 & 0 & i
\end{array}\right),
$$


which produces a two-parameter family of solutions

$$
u\left(t ; \theta_{1}, \theta_{2}\right)=G\left(\theta_{1}\right)\left(A\left(t+\theta_{2}\right), N\left(t+\theta_{2}\right), B\left(t+\theta_{2}\right)\right)
$$

from any solution of (50)-(52). In a continuation context, when looking for the next solution on a branch of solutions, we want to find the one closest in norm to the previous solution $u^{(0)}$. Hence, the new solution $u$ is chosen to minimize

$$
\left.D\left(\theta_{1}, \theta_{2}\right)=\int_{0}^{1} \| u\left(t ; \theta_{1}, \theta_{2}\right)-u^{(0)}(t)\right) \|_{2}^{2} \mathrm{~d} t
$$

in $\theta_{1}, \theta_{2}$. Differentiating with respect to both variables and evaluating at $\theta_{1}=$ $\theta_{2}=0$ yields

$$
\begin{gathered}
\int_{0}^{1} \dot{u}^{(0)}(s)\left(u^{(0)}(s)-u(s)\right) \mathrm{d} s=0 \\
\int_{0}^{1} \mathfrak{G} u^{(0)}(s)\left(u^{(0)}(s)-u(s)\right) \mathrm{d} s=0,
\end{gathered}
$$

where $\mathfrak{G}=\mathrm{d} /\left.\mathrm{d} \theta G(\theta)\right|_{\theta=0}$ is the infinitesimal generator of the symmetry group. Note that (53) is actually the phase condition (14), while (54) is a new phase condition that fixes the group invariance.

With (53) and (54) periodic solutions can be continued as isolated solutions. These phase conditions are implemented in both DDE-BIFTOOL and PDDE-Cont. In [29] DDE-BIfTool was used to compute the periodic solutions of (50)-(52) and PDDE-CONT was used to determine their stability boundraries by continuing the Neimark-Sacker bifurcation in two parameters.

The resulting stability regions of the two different types of periodic solutions are colored in Fig. 12(a), and examples of typical time series are shown in panels (b)-(d). First, there are the typical relaxation oscillations (not to be confused with relaxation oscillations of slow-fast systems as discussed in Chapter 8), which are a periodic exchange of energy between the electric field $E$ and the inversion $N$ in a semiconductor lasers. Relaxation oscillations are fast (on the order of a few $\mathrm{GHz}$ ) and effectively do not involve the filter; see Fig. 12(b). The other type of oscillations are the frequency oscillations, which are slower and oscillate on the time scale given by the external roundtrip time (that is, the delay $\tau$ ); see Fig. 12(c) and (d). These oscillations are unusual for semiconductor lasers because they feature practically constant laser intensity $I_{L}$ but an oscillating frequency $\omega_{L}$. Notice that the dynamics of the filter appears to suppress the dynamics of the intensity. Both types of oscillations lose their stability at Neimark-Sacker bifurcations, which are shown as black curves in Fig. 12(a). 


\section{Conclusions}

We discussed numerical continuation methods for the stability and bifurcation analysis of delay differential equations with constant delays, concentrating on techniques concerning steady-state solutions and periodic solutions. We also described how to compute connecting (homoclinic and heteroclinic) orbits and quasiperiodic solutions. Furthermore, wqe briefly mentioned how to deal with state dependent delays and with equations of neutral type. Compared with numerical methods for such tasks in ordinary differential equations the methods we presented are either similar but with a higher computational cost (an example is collocation for computing periodic solutions) or much more complex (as is the case for computing the stability of a steady state or finding connected orbit). These additional difficulties are due to the infinitedimensional nature of DDEs.

Rather than trying to give a complete literature survey, we focused on the numerical methods implemented in the software packages DDE-BIFTOOL and PDDE-CONT. Both have about the same functionality as similar packages for ODEs, but with less flexibility and at a higher computational cost. They make continuation and bifurcation analysis for DDEs readily available for scientists dealing with concrete problems arising in applications. We have included results on the continuation and bifurcation analysis of several realistic models to illustrate the applicability of the methods.

Numerical developments can also help with the solution of some open theoretical problems. For example, some numerical results on state-dependent DDEs are ahead of the theory and suggest that certain conditions imposed in the theory are rather technical and not fundamental. One of the areas for future work for both theory and numerical methods is that of piecewisesmooth delayed systems, which have important applications, for example, in control theory $[4,68]$, hybrid testing $[51,69]$ and machining $[22, ?]$.

\section{Acknowledgements}

We thank Bernd Krauskopf, David Barton, Tatyana Luzyanina, Giovanni Samaey and Koen Verheyden for their useful comments and suggestions. We are grateful to the American Physical Society, World Scientific Publishing and the Society for Industrial and Applied Mathematics for permission to reproduce Fig. 4, Fig. 5 and Fig. 12, respectively.

\section{References}

1. U. M. Ascher, R. M. M. Mattheij, and R. D. Russell. Numerical Solution of Boundary Value Problems for Ordinary Differential Equations. (Prentice Hall, 1988). 
2. C. T. H. Baker, C. A. H. Paul, and D. R. Willé. A bibliography on the numerical solution of delay differential equations. Technical Report 269, University of Manchester, Manchester Centre for Computational Mathematics, 1995.

3. B. Balachandran. Non-linear dynamics of milling process. Trans. Royal Soc., 359:793-820, 2001.

4. D. A. W. Barton, B. Krauskopf, and R. E. Wilson. Explicit periodic solutions in a model of a relay controller with delay and forcing. Nonlinearity, 18(6):26372656, 2005.

5. D. A. W. Barton, B. Krauskopf, and R. E. Wilson. Collocation schemes for periodic solutions of neutral delay differential equations. J. Diff. Eqns. Appl., 12(11):1087-1101, 2006.

6. A. Bellen and M. Zennaro. Numerical Methods for Delay Differential Equations. (Oxford University Press, 2003).

7. W. J. Beyn. The numerical computation of connecting orbits in dynamical systems. IMA J. Numer. Analysis, 9:379-405, 1990.

8. W. J. Beyn, A. Champneys, E. J. Doedel, W. Govaerts, B. Sandstede, and Y. A. Kuznetsov. Numerical continuation and computation of normal forms. In B. Fiedler, editor, Handbook of Dynamical Systems, pages 149-219. (Elsevier, 2002).

9. D. Breda. Numerical computation of characteristic roots for delay differential equations. $\mathrm{PhD}$ thesis (Department of Mathematics and Computer Science, University of Udine, 2004).

10. D. Breda. Solution operator approximation for delay differential equation characteristic roots computation via Runge-Kutta methods. Appl. Numer. Math., 56:305-317, 2006.

11. D. Breda, S. Maset, and R. Vermiglio. Computing the characteristic roots for delay differential equations. IMA J. Numer. Analysis, 24:1-19, 2004.

12. D. Breda, S. Maset, and R. Vermiglio. Pseudospectral differencing methods for characteristic roots of delay differential equations. SIAM J. Sci. Comput., 27(2):482-495, 2005.

13. A. M. Castelfranco and H. W. Stech. Periodic solutions in a model of recurrent neural feedback. SIAM J. Appl. Math., 47(3):573-588, 1987.

14. K. L. Cooke and W. Huang. On the problem of linearization for state-dependent delay differential equations. Proc. Am. Math. Soc., 124(5), 1996.

15. S. P. Corwin, D. Sarafyan, and S. Thompson. DKLAG6: A code based on continuously imbedded sixth order Runge-Kutta methods for the solution of state dependent functional differential equations. Appl. Numer. Math., 24(23):319-330, 1997.

16. T. A. Davies. UMFPACK Version 4.1 User Guide. Technical report, Department of Computer and Information Science and Engineering, University of Florida, Gainesville, FL, USA, 2003. http://www.cise.ufl.edu/research/sparse/ umfpack/.

17. E. J. Doedel, A. R. Champneys, T. F. Fairgrieve, Y. A. Kuznetsov, B. Sandstede, and X. Wang. AUTO97: Continuation and bifurcation software for ordinary differential equations, 1997; available via ftp.cs.concordia.ca in directory pub/doedel/auto.

18. E. J. Doedel, M. J. Friedman, and B. I. Kunin. Successive continuation for locating connecting orbits. Num. Alg., 17:103-124, 1997. 
19. E. J. Doedel, W. Govaerts, and Yu. A. Kuznetsov. Continuation of periodic solution bifurcations in odes using bordered systems. SIAM J. Numer. Analysis, 42(2):401-435, 2003.

20. E. J. Doedel, H. B. Keller, and J. P. Kernevez. Numerical analysis and control of bifurcation problems (II): bifurcations in infinite dimensions. Internat. J. Bifur. Chaos Appl. Sci. Engrg., 1(4):745-772, 1991.

21. E. J. Doedel and P. P. C. Leung. A numerical technique for bifurcation problems in delay differential equations. Congr. Numer., 34:225-237, 1982. (Proc. 11th Manitoba Conf. Num. Math. Comput., Univ. Manitoba, Winnipeg, Canada).

22. Z. Dombóvári. Bifurcation analysis of a cutting process. Master's thesis (Budapest University of Technology and Economics, 2006).

23. K. Engelborghs and E. Doedel. Stability of piecewise polynomial collocation for computing periodic solutions of delay differential equations. Numer. Math., 91(4):627-648, 2002.

24. K. Engelborghs, T. Luzyanina, K. J. in 't Hout, and D. Roose. Collocation methods for the computation of periodic solutions of delay differential equations. SIAM J. Sci. Comput., 22(5):1593-1609, 2000.

25. K. Engelborghs, T. Luzyanina, and D. Roose. Numerical bifurcation analysis of delay differential equations using DDE-BIFTOOL. ACM Trans. Math. Software, 28(1):1-21, 2002.

26. K. Engelborghs, T. Luzyanina, and G. Samaey. DDE-BIFTOOL v. 2.00: a Matlab package for bifurcation analysis of delay differential equations. Technical Report TW-330, Department of Computer Science, K.U.Leuven, Leuven, Belgium, 2001; available at www.cs.kuleuven.ac.be/cwis/research/ ${ }^{\sim}$ twr/research/ software/delay/ddebiftool. shtml.

27. K. Engelborghs and D. Roose. On stability of LMS methods and characteristic roots of delay differential equations. SIAM J. Numer. Analysis, 40(2):629-650, 2002.

28. B. Ermentrout. XPPAUT3.91 - The differential equations tool. University of Pittsburgh, Pittsburgh, 1998; available at http://www.pitt.edu/ phase/.

29. H. Erzgräber, B. Krauskopf, and D. Lenstra. Bifurcation analysis of a semiconductor laser with filtered optical feedback. SIAM J. Appl. Dyn. Sys., 6(1):??-??, 2007.

30. W. Govaerts. Bordered augmented linear systems in numerical continuation and bifurcation. Numer. Math., 58:353-368, 1990.

31. W. Govaerts and J. D. Pryce. Mixed block elimination for linear systems with wider borders. IMA J. of Numer. Analysis, 13:161-180, 1993.

32. K. Green, B. Krauskopf, and K. Engelborghs. Bistability and torus break-up in a semiconductor laser with phase-conjugate feedback. Physica D, 173(1-2):114$129,2002$.

33. K. Green, B. Krauskopf, and K. Engelborghs. One-dimensional unstable eigenfunction and manifold computations in delay differential equations. J. Comp. Phys., 197(1):86-98, 2004.

34. K. Green, B. Krauskopf, and G. Samaey. A two-parameter study of the locking region of a semiconductor laser subject to phase-conjugate feedback. SIAM J. Appl. Dyn. Sys., 2(2):254-276, 2003.

35. B. Haegeman, K. Engelborghs, D. Roose, D. Pieroux, and T. Erneux. Stability and rupture of bifurcation bridges in semiconductor lasers subject to optical feedback. Phys. Rev. E, 66:046216 1-11, 2002. 
36. J. K. Hale. Theory of Functional Differential Equations, volume 3 of Applied Mathematical Sciences. (Springer-Verlag, New York, 1977).

37. J. K. Hale and N. Sternberg. Onset of chaos in differential delay eqautions. $J$. Comp. Phys., 77:221-239, 1988.

38. J. K. Hale and S. M. Verduyn Lunel. Introduction to Functional Differential Equations, volume 99 of Applied Mathematical Sciences. (Springer-Verlag, New York, 1993).

39. F. Hartung and J. Turi. Stability in a class of functional differential equations with state-dependent delay. In C Corduneanu, editor, Qualitative problems for differential equations and control theory, pages 15-31. (World Scientific, Singapore, 1995).

40. F. Hartung and J. Turi. On differentiability of solutions with respect to parameters in state-dependent delay equations. J. Diff. Eqns., 135:192-237, 1997.

41. B. D. Hassard. A code for Hopf bifurcation analysis of autonomous delaydifferential systems. In F. V. Atkinson, W. F. Langford, and A. B. Mingarelli, editors, Oscillations, Bifurcations and Chaos, volume 8 of Can. Math. Soc. Conference Proceedings, pages 447-463 (Amer. Math. Soc., Providence, RI, 1987).

42. B. D. Hassard. Counting roots of the characteristic equation for linear delaydifferential systems. J. Diff. Eqns., 136:222-235, 1997.

43. J. S. Hesthaven. Spectral penalty methods. Appl. Num. Math., 33:23-41, 2000.

44. K. J. in 't Hout and Ch. Lubich. Periodic orbits of delay differential equations under discretization. BIT, 38(1):72-91, 1998.

45. T. Insperger, B. P. Mann, G. Stépán, and P. V. Bayly. Stability of up-milling and down-milling, part 1: alternative analytical methods. Int. J. of Mach. Tool Manuf., 43(1):25-34, 2003.

46. M. A. Kaashoek and S. M. Verduyn Lunel. Characteristic matrices and spectral properties of evolutionary systems. Trans. Amer. Math. Soc., 334:479-517, 1992.

47. D.M. Kane and K.A. Shore (Eds.), Unlocking Dynamical Diversity: Optical Feedback Effects on Semiconductor Lasers, (John Wiley \& Sons, 2005).

48. D. A. Kopriva and J. H. Kolias. A conservative staggered-grid chebyshev multidomain method for compressible flows. J. Comp. Phys., 125:244-261, 1996.

49. B. Krauskopf. Bifurcation analysis of lasers with delay. In D.M. Kane and K.A. Shore, editors, Unlocking Dynamical Diversity: Optical Feedback Effects on Semiconductor Lasers, pages 147-183. (John Wiley \& Sons, 2005).

50. Yu. A. Kuznetsov and V. V. Levitin. CONTENT: A multiplatform environment for analyzing dynamical systems, 1997. Dynamical Systems Laboratory, Centrum voor Wiskunde en Informatica; available via ftp.cwi.nl in directory urlpub/CONTENT.

51. Y. N. Kyrychko, K. B. Blyuss, A. Gonzalez-Buelga, S. J. Hogan and D. J. Wagg. Real-time dynamic substructuring in a coupled oscillator-pendulum system. Proc. R. Soc. A 462:1271-1294, 2006.

52. K. Lust, D. Roose, A. Spence, and A.R. Champneys. An adaptive NewtonPicard algorithm with subspace iteration for computing periodic solutions. SIAM J. Sci. Comput., 19(4):1188-1209, 1998.

53. T. Luzyanina and K. Engelborghs. Computing Floquet multipliers for functional differential equations. Internat. J. Bif. Chaos Appl. Sci. Engrg., 12(12):29772989, 2002.

54. T. Luzyanina and D. Roose. Numerical stability analysis and computation of Hopf bifurcation points for delay differential equations. J. Comput. Appl. Math., 72:379-392, 1996. 
55. M. C. Mackey and L. Glass. Oscillation and chaos in physiological control systems. Science, 77:287-289, 1977.

56. J. Mallet-Paret and R. D. Nussbaum. A differential delay equation arising in optics an physiology. SIAM J. Math. Analysis, 20(2):249-292, 1989.

57. J. Mallet-Paret and R.D. Nussbaum. Boundary layer phenomena for differentialdelay equations with state-dependent time lags 1. Arch. Rational Mech. Analysis, 120:99-146, 1992.

58. S. I. Niculescu. Delay Effects on Stability: A Robust Control Approach. Lecture Notes in Control and Information Science, 269, (Springer-Verlag, New York, 2000).

59. M. Nizette and T. Erneux. Optical frequency dynamics and relaxation oscilations of a semiconductor laser subject to filtered optical feedback. In M. Pessa D. Lenstra and Ian H White, editors, Semiconductor Lasers and Laser Dynamics II, Proceedings of the SPIE, pages 6184-32. 2006.

60. G. Orosz, B. Krauskopf, and R. E. Wilson. Bifurcations and multiple traffic jams in a car following model with reaction delay time. Physica D, 211:277-293, 2005.

61. G. Orosz, R. E. Wilson, and B. Krauskopf. Global bifurcation investigation of an optimal velocity traffic model with driver reaction time. Phys. Rev. E, 70(026207):1-10, 2004.

62. C. A. H. Paul. A user-guide to Archi - an explicit Runge-Kutta code for solving delay and neutral differential equations. Technical Report 283 (The University of Manchester, Manchester Center for Computational Mathematics, 1997).

63. A. Pazy. Semigroups of linear operators and applications to Partial differential equations. (Springer-Verlag, New York, 1983).

64. Y. Saad. Iterative Methods for Sparse Systems. (SIAM, Philadelphia, 2003).

65. G. Samaey, K. Engelborghs, and D. Roose. Numerical computation of connecting orbits in delay differential equations. Num. Algor., 30(3-4):335-352, 2002.

66. G. Samaey and B. Sandstede. Determining stability of pulses for partial differential equations with time delays. Dyn. Sys., 20(2):201-222, 2005.

67. F. Schilder, H. M. Osinga, and W. Vogt. Continuation of quasiperiodic invariant tori. SIAM J. Appl. Dyn. Sys., 4(3):244-261, 2005.

68. J. Sieber. Dynamics of delayed relay systems. Nonlinearity, 19(11):2489-2527, 2006.

69. J. Sieber and B. Krauskopf. Control-based bifurcation analysis for experiments. Nonlin. Dyn. in press.

70. G. Stépán. Retarded Dynamical Systems. (Longman, London, 1989).

71. G. Stépán and T. Kalmár-Nagy. Nonlinear regenerative machine tool vibrations. In Proceedings of the 1997 ASME Design Engineering Technical Conferences, Sacramento, California, September 1997. Paper number DETC97/VIB-4021.

72. G. Stépán, R. Szalai, and T. Insperger. Stability of high-speed milling. In G. Radons, editor, Nonlinear Dynamics of Production Systems, pages 111-128. (Wiley VCH, 2003).

73. R. Szalai. PDDE-CONT: A continuation and bifurcation software for delaydifferential equations, 2005. Department of Applied Mechanics, Budapest University of Technolgy and Economics; available at http://www.mm.bme.hu/ szalai/pdde/.

74. R. Szalai, B. P. Mann, and G. Stépán. Period-two and quasiperiodic vibrations of high-speed milling. In I. Grabec and E. Govekar, editors, Proceedings of the 9th CIRP International Workshop on Modeling of Machining Operations, pages 107-114. CIRP, 2006. 
75. R. Szalai, G. Stépán, and S. J. Hogan. Continuation of bifurcations in periodic delay-differential equations using characteristic matrices. SIAM J. Sci. Comp., 28(4):1301-1317, 2006.

76. L. N. Trefethen. Spectral methods in MATLAB. (SIAM, Philadelphia, 2000).

77. K. Verheyden, K. Green, and D. Roose. Numerical stability analysis of a largescale delay system modeling a lateral semiconductor laser subject to optical feedback. Phys. Rev. E, 69(3):036702, 2004.

78. K. Verheyden and K. Lust. A Newton-Picard collocation method for periodic solutions of delay differential equations. BIT Num. Math., 45(3):1605-625, 2005.

79. K. Verheyden, T. Luzyanina, and D. Roose. Efficient and reliable stability analysis of solutions of delay differential equations. Proceedings of 2006 International Conference on Nonlinear Science and Complexity, pages 109-120. (World Scientific Publishing, Singapore, 2006).

80. K. Verheyden, T. Luzyanina, and D. Roose. Efficient computation of characteristic roots of delay differential equations using LMS methods. J. Comp. Applied Math., 2006. submitted.

81. K. Verheyden and D. Roose. Efficient numerical stability analysis of delay equations: a spectral method. In D. Roose and W. Michiels, editors, Time-delay systems 2004, IFAC Proceedings Volumes, pages 209-214. (Elsevier, Oxford, UK, 2005). 